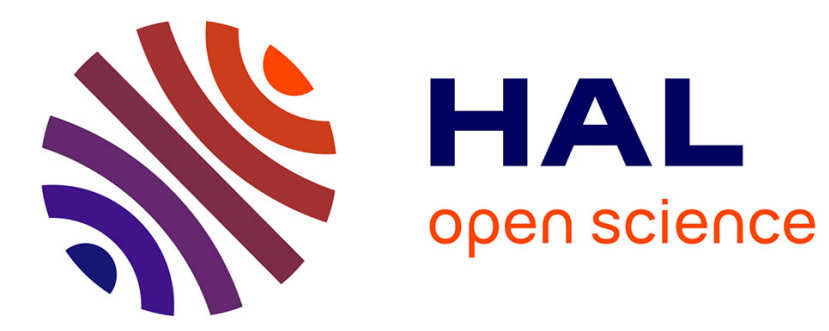

\title{
Measuring Conflicts of Multisource Imprecise Information in Multistate System Reliability Assessment
}

Tangfan Xiahou, Zhiguo Zeng, Yu Liu, Hong-Zhong Huang

\section{To cite this version:}

Tangfan Xiahou, Zhiguo Zeng, Yu Liu, Hong-Zhong Huang. Measuring Conflicts of Multisource Imprecise Information in Multistate System Reliability Assessment. IEEE Transactions on Reliability, 2021, pp.1-18. 10.1109/TR.2021.3087531 . hal-03464076

\section{HAL Id: hal-03464076 https://hal.science/hal-03464076}

Submitted on 11 Jan 2022

HAL is a multi-disciplinary open access archive for the deposit and dissemination of scientific research documents, whether they are published or not. The documents may come from teaching and research institutions in France or abroad, or from public or private research centers.
L'archive ouverte pluridisciplinaire HAL, est destinée au dépôt et à la diffusion de documents scientifiques de niveau recherche, publiés ou non, émanant des établissements d'enseignement et de recherche français ou étrangers, des laboratoires publics ou privés. 


\title{
Measuring Conflicts of Multi-Source Imprecise Information in Multi-State System Reliability Assessment
}

\author{
Tangfan Xiahou, Zhiguo Zeng, Yu Liu, Senior Member, IEEE, and Hong-Zhong Huang
}

\begin{abstract}
In engineering scenarios, expert judgements play an essential role in reliability assessment, especially for those systems with few historical data. To achieve a rational result, experts from different areas should be involved, and the uncertainties in their assessments should be properly addressed. Such information is often referred to as multi-source imprecise information (MSII) and might contain high degree of conflicts, as different experts usually have different expertise and knowledge. Properly quantifying the conflicts among the MSII, then, becomes a critical issue, as the subsequent processing of MSII (e.g., combination and calibration), depends on the degree of conflict in the MSII. To this end, a new conflict measure is put forth based on the DempsterShafer theory (DST) to quantify and visualize the conflict in the MSII from a group of experts. In the first place, the MSII from each expert is used to construct the basic belief assignment (BBA) of the reliability estimates for the corresponding expert under the DST. A two-dimensional conflict measure, which combines the conflict factor and Jousselme distance in DST, is, then, proposed to measure the conflict between the experts' BBAs. The conflict is quantified from two perspectives, viz., mutual conflict and total conflict. Finally, a Bhattacharyya distance-based method is developed to further quantify the informativeness of each expert's MSII to the system reliability estimate. A numerical example along with an engineering case is used to validate the effectiveness of the proposed approach.
\end{abstract}

Index Terms - multi-state system (MSS), conflict measure, multi-source imprecise information (MSII), Dempster-Shafer theory, Bhattacharyya distance.

\section{NOMENCLATURE}

MSS Multi-State System

MSII Multi-Source Imprecise Information

DST Dempster-Shafer Theory

FoD Frame of Discernment

BBA Basic Belief Assignment

MTTF Mean Time To Failure

CRC Conjunctive Rule of Combination

This work was support by the National Natural Science Foundation of China under contact numbers 71922006 and 71771039. (Corresponding author: Yu Liu)

T. Xiahou is with the School of Mechanical and Electrical Engineering, University of Electronic Science and Technology of China, Chengdu, Sichuan 611731, China. (e-mail: xiahoutf@163.com)

Z. Zeng is with Chaire on Risk and Resilience of Complex Systems, Université Paris Saclay, CentraleSupélec, Laboratoire Genie Industriel, Gifsur-Yvette, France. (e-mail: zhiguo.zeng@ @entralesupelec.fr)

Y. Liu and H. Z. Huang are with the School of Mechanical and Electrical Engineering and also with the Center for System Reliability and Safety, University of Electronic Science and Technology of China, Chengdu, Sichuan 611731, China. (e-mail: yuliu@uestc.edu.cn, hzhuang@uestc.edu. cn.)
DRC

$R_{S}(t)$

$R_{S L, k}(t)$

$R_{S U, k}(t)$

$R_{S L}(t)$

$R_{S U}(t)$

$\mathbf{m}_{k}(t)$

$k_{i \oplus j}$

$J D\left(\mathbf{m}_{i}, \mathbf{m}_{j}\right)$

$k_{\mathbf{m}_{i} \oplus \mathbf{m}_{\oplus \bar{i}}}$

$J D\left(\mathbf{m}_{i}, \mathbf{m}_{\oplus \bar{i}}\right)$

$\delta_{B}\left(q_{R_{S U}(t)}, q_{R_{S L}(t)}\right)$

$\delta_{B}\left(q_{R_{S U, \bar{k}}(t)}, q_{R_{S L, \bar{k}}(t)}\right)$ distributions without the $k$ th expert's MSII

\section{INTRODUCTION}

A DVANCED engineering systems are getting everincreasingly complex and sophisticated nowadays. Traditional system reliability models, which are binary-state (failed/working) in nature, are not able to characterize the complicated deterioration profiles arising from the increasing system complexity. The complex degradation profiles can be considered by multi-state system (MSS) reliability models, as they introduce intermediate states between perfectly working and completely failed state. MSS reliability models have, therefore, received tremendous attention in the past a few decades [1]-[3]. In engineering practices, many systems execute their planned missions with multiple levels of damage severities or performance capacities. These systems can be naturally modelled as MSSs. As MSSs are more complicated than binarystate systems, novel methods are needed for their reliability assessment, e.g., universal generating function (UGF) [4], 
multi-valued decision diagram [5], stochastic processes [6], and simulation-based methods [7].

Most of the researches on reliability assessment of MSSs are based on the premise that the state probabilities of an MSS and its components can be precisely known, through by rigorous statistical estimation based on a large amount of test/event data. In practice, however, due to the limited reliability testing resources (e.g., time, budget, manpower), the collected test/event data are often scarce and cannot satisfy the largesample-size requirement for rigorous statistical analyses. Traditional MSS reliability assessment methods are, therefore, unable to handle such a challenge. Expert judgements are often used, as an alternative to statistical data, to assess the state probabilities under such scenarios. Typical procedures of reliability assessment using expert judgements contains three steps: elicitation of expert judgements, combination of expert judgements, and calibration of expert judgements [8]. The latter two steps are oftentimes carried out in an interactive manner in order to achieve a rational consensus among a group of experts on the elicited information.

Expert judgement elicitation is a process where experts are asked to express their judgements and the associated uncertainties. Typical approaches to represent the uncertainty include Bayesian approach [9], interval set theory [10], Dempster-Shafer theory (DST) [11],[12], and fuzzy set theory [13]. For example, Ding and Lisnianski [13] defined the fuzzy MSSs (FMSSs) and investigated a fuzzy universal generating function (FUGF)-based approach to calculate the fuzzy reliability of an MSS, where the performance capacity and state probability of components were elicited from experts and modelled by triangular fuzzy numbers. The FUGF-based reliability assessment of FMSSs was extended by Liu and Huang [14] into a fuzzy Markov model. Parametric programming was used to assess the instantaneous fuzzy state probabilities of MSSs. Under the interval set theory, Li et al. [15] developed an affined interval UGF (AIUGF) method to evaluate the stationary state probabilities of MSSs when the state probabilities of components were elicited as interval values. Under the DST, Destercke and Sallak [16] represented the state probabilities of components as mass functions and used several combination rules to evaluate the MSS reliability. Their method can effectively handle the credibility of expert judgements and their interdependences. Nonetheless, all the above methods can be applied when only the imprecise state probability of components need to be elicited. In engineering scenarios, many MSSs may exhibit a hierarchical structure in which the system can be decomposed into many subsystems and each subsystem can contain more than one component. The imprecise expert judgements need, therefore, be elicited on multiple levels of an MSS, e.g., component-level, subsystemlevel, and system-level. On the other hand, as experts may possess distinct knowledge backgrounds, the imprecise expert judgements are heterogeneous in nature. Further, different experts might provide imprecise information regarding the system reliability at distinct time instants. If expert judgement possesses the three foregoing characteristics, it is defined as multi-source imprecise information (MSII) [10].
After eliciting expert judgements, the expert judgements are combined for system reliability assessment, the results also indicate whether the reliability estimate is satisfactory. If not, we need to calibrate the expert judgements [28]. Specifically, by taking account of the aforementioned MSII, system reliability bounds were assessed in our earlier work [10] by formulating a pair of constrained optimization models. In the constrained optimization models, the optimization objective was the system reliability function, whereas the constraints were constructed to represent the MSII from the experts as functions of the unknown parameters. By maximizing and minimizing the system reliability function under these constraints, the upper and lower bounds of system reliability were estimated over time. As observed from our earlier work, the resulting reliability bounds from different experts can vary from one another. In other words, the reliability bounds constructed individually based on each expert's MSII might conflict partially or completely with each other. When the reliability bounds were not completely conflicting, the narrowest reliability bound which satisfies all the constraints should be selected as the final result. In contrast, when reliability bounds from the MSII of a group of experts have no intersections, the combination of expert judgements cannot reach consensus on the reliability estimate. Calibration of expert judgements should, then, be carried out [28].

Calibration of expert judgments is not trivial as decision makers should decide which one (ones) of the expert judgement(s) need to be calibrated and to what extent [8]. To facilitate such choices, it is of great significance to quantitatively measure and visualize the conflict and/or consistency among experts' judgements, as well as the informativeness of each expert judgment with respect to estimating the system reliability. An illustration of the reliability assessment of an apogee engine is provided to show the importance of measuring the conflict and informativeness of MSII from experts. The apogee engine intends to complete the mission of starting at apogee and sending the satellite into the synchronous orbit [27]. To ensure the success of the mission, multiple domain experts with different knowledge backgrounds are invited to elicit their knowledge for the reliability assessment of the apogee engine. The engine mainly consists of three parts: an ignition structure, an engine shell, and a nozzle. The ignition structure can be further decomposed into three kinds of components, namely, an igniter, two spark plugs, and an ignition composition. The ignition composition and nozzle are innovative products with few failure data, only the bounds of the mean time to failure (MTTF) of these two components are elicited from two experts, while the reliability bounds of the subsystems, i.e., the ignition structure, engine shell, and the propellant grain are, respectively, elicited from another three experts. The experts' imprecise information is, therefore, a typical type of MSII. However, as both the bound of MTTF of the ignition composition and the reliability bound of the ignition structure are information related to the ignition structure, these two pieces of information may be quite conflicting as they come from two different experts. In order to achieve a consensus on the reliability assessment of the entire 
apogee engine, it, therefore, necessitates manipulate the conflict quantification among the experts' information in order to decide which is to be calibrated or refined. Meanwhile, the informativeness of the experts' MSII is also critical to the refinement of the experts, that is, to decide whom to keep and whom to get rid of.

DST is a natural and widely accepted non-probabilistic framework for such task [25]. There are numerous ways to quantify conflicts among evidences from experts under the DST. Dempster is the pioneer to provide the definition of conflicts and used the conflict factor for their quantification [19]. Schubert [21] used the degree of falsity for conflict management of evidences. Destercke and Burger [22] defined an interval-valued conflict measure based on the contour functions in DST to measure the disagreement between evidences. Many researches used distance measures, such as Jousselme distance [23], cosine measure [24], and Minkowski measures [25], to quantify the conflict between evidences. It bears noting that even though a plethora of research efforts have been devoted to quantifying the conflict among experts' evidences under the DST, these conflict measures can merely tackle homogeneous evidences, which is referred to as the evidences that are elicited from the same frame of discernment (FoD) at the same time instants [22]. They cannot be directly implemented on MSII: MSII is inhomogeneous, since it involves hierarchical structures which are defined in different FoDs and can be given at distinct time instants during the system's life cycle.

To fill the aforementioned research gaps, this article aims at developing a framework to quantify the conflict among the MSII of a group of experts for reliability assessment. Firstly, the different MSII is unified by transforming then into the reliability of MSSs. This is mainly done by using the reliability bound assessment method proposed in our previous work [10]. Secondly, we use the reliability bound induced from each expert's MSII to derive their corresponding basic belief assignments (BBAs) via the interval-to-mass transformation [17]. Therefore, the experts' MSII are converted into a set of evidences about the reliability of the MSSs. Thirdly, a twodimensional conflict measure, which combines the conflict factor [19] and Jousselme distance [23] in DST, is proposed to measure the conflicts among these pieces of evidences. We quantify the conflicts from two perspectives, viz., mutual conflict and total conflict. Mutual conflict is defined as the conflict between any two pieces of evidences whereas total conflict is defined as the conflict of a specific piece of evidence with respect to all the other evidences. The mutual conflict is quantified by applying the two-dimensional conflict measure on each pair of the experts, while the total conflict quantification considers the conflict of each expert's evidence against aggregated evidence, constructed by combining the rest of the experts via Dempster's rule of combination (DRC). Lastly, in order to further quantify the informativeness of a specific expert's MSII, a Bhattacharyya distance-based informativeness measure is put forth. Two examples, a multi-state flow transmission system and a cutter feeding control system, are presented to validate the developed framework.

The remainder of this article is structured as follows. Section
II offers a brief review of the MSS and the reliability bound assessment approach developed in our earlier work. A twodimensional conflict measure is put forth in Section III and a framework of conflict quantification among experts' MSII by the two-dimensional conflict measure is presented in Section IV. Section V presents a Bhattacharyya distance-based measure to quantify the informativeness of each expert. The effectiveness of the proposed method is demonstrated in Section VI via a numerical example along with an engineering application of the cutter feeding control system of CNC lathes. Section VII is the conclusion and a summary of future works.

\section{Multi-State System ReliabILITY MODEL AND MSII}

\section{A. Multi-State Systems}

In this article, we consider an MSS with a hierarchical structure, that is, the system can be decomposed into subsystems and each subsystem contains at least one multi-state component. MSII can be collected from all of these hierarchies, i.e., it can be collected from the system level, the subsystem level, and the component level. In practice, the state in the MSS can be used to describe performance capacity or damage severity that has discrete levels. The state of the subsystem and system can be determined by the corresponding structure functions.

A flow transmission system, delineated in Fig. 1, is used for the illustration of an MSS. The flow transmission system is composed of three pipes, in which Pipes \#1 and \#2 are connected in parallel and then serially connected to Pipe \#3. Pipes \#1 and \#2 can be viewed as a subsystem, denoted as $S_{1}$. The performance capacity of the system and its components is measured by the transmission capacity (tons/min). Pipes \#1 and \#2 are assumed to have three states, where State 0 represents complete failure corresponding to a capacity of 0 ; State 2 manifests a full capacity, and State 1 represents a partial failure. Pipe \#3 has only two states: State 0 represents failure and State 1 represents working. The capacities of all the components are tabulated in Table I. Based on the system configuration, both subsystem $S_{1}$ and the whole flow transmission system can exhibit seven states, as tabulated in Table II and Table III, respectively.

Without loss of generality, the MSS can be defined based on the following assumptions:

(1) An MSS contains $M_{C}$ components, which are grouped into $M_{\text {sub }}$ subsystems. Component $l\left(l=1,2, \ldots, M_{C}\right)$ possesses a finite number of states, denoted by $0,1,2, \ldots, N_{C_{1}}$. Due to the multi-state nature of components, subsystem $m$ ( $\left.m=1,2, \ldots, M_{\text {sub }}\right)$ and the entire system also manifest multiple states, denoted by $0,1,2, \ldots, N_{S_{m}}$ and $0,1,2, \ldots, N_{S}$, respectively. Among these states, State 0 is the worst state, whereas the states $N_{C_{l}}, N_{S_{m}}$, and $N_{S}$ represent the states with best performances, respectively.

(2) To quantitatively characterize the behaviors of the components of the studied system, the stochastic behavior of component $l \quad\left(l \in\left\{1,2, \ldots, M_{C}\right\}\right)$ is characterized by homogenous discrete-state continuous-time Markov models 
[6]. For the homogenous Markov model of component $l$, the transition intensity $\lambda_{l,(i, i)}(j<i)$ from state $i$ to state $j$ is a

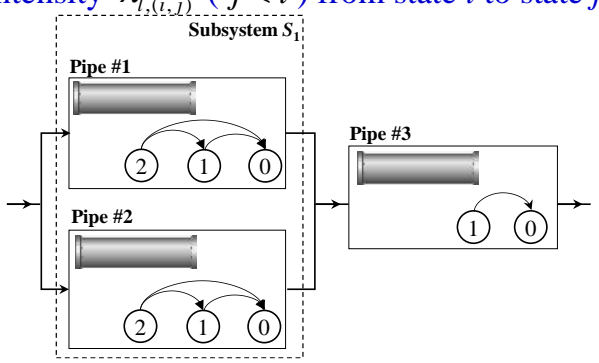

Fig. 1. The configuration of the flow transmission system [10].

TABLE I

THE PERFORMANCE CAPACITY OF ALL THE COMPONENTS

\begin{tabular}{cccc}
\hline \hline Pipe ID & State 2 & State 1 & State 0 \\
\hline$\# 1$ & 1.5 tons $/ \mathrm{min}$ & 1.0 tons $/ \mathrm{min}$ & 0.0 tons $/ \mathrm{min}$ \\
$\# 2$ & 2.0 tons $/ \mathrm{min}$ & 1.5 tons $/ \mathrm{min}$ & 0.0 tons $/ \mathrm{min}$ \\
$\# 3$ & - & 4.0 tons $/ \mathrm{min}$ & 0.0 tons $/ \mathrm{min}$ \\
\hline \hline
\end{tabular}

TABLE II

THE STATES OF SUBSYSTEM $S_{1}$ AND ITS CORRESPONDING STATE COMBINATIONS OF PIPES \#1 AND \#2.

\begin{tabular}{|c|c|c|c|}
\hline $\begin{array}{c}\text { State of } \\
\text { subsystem } S_{1}\end{array}$ & $\begin{array}{c}\text { Performance } \\
\text { capacity }\end{array}$ & $\begin{array}{l}\text { State of } \\
\text { Pipe \#1 }\end{array}$ & $\begin{array}{l}\text { State of } \\
\text { Pipe \#2 } \\
\end{array}$ \\
\hline 0 & \multicolumn{2}{|l|}{0.0 tons $/ \mathrm{min}$} & 0 \\
\hline 1 & \multicolumn{2}{|l|}{1.0 tons $/ \mathrm{min}$} & 0 \\
\hline \multirow{2}{*}{2} & \multirow{2}{*}{\multicolumn{2}{|c|}{1.5 tons $/ \mathrm{min}$}} & 1 \\
\hline & & 2 & 0 \\
\hline 3 & \multicolumn{2}{|l|}{2.0 tons $/ \mathrm{min}$} & 2 \\
\hline 4 & \multicolumn{2}{|l|}{2.5 tons $/ \mathrm{min}$} & 1 \\
\hline \multirow{2}{*}{5} & \multirow{2}{*}{3.0 tons $/ \mathrm{min}$} & 1 & 2 \\
\hline & & 2 & 1 \\
\hline 6 & \multicolumn{2}{|l|}{3.5 tons $/ \mathrm{min}$} & 2 \\
\hline \multicolumn{4}{|c|}{$\begin{array}{c}\text { TABLE III } \\
\text { THE SYSTEM STATES AND ITS CORRESPONDING STATE } \\
\text { COMBINATIONS OF SUBSYSTEM } S_{1} \text { AND PIPE \#3. } \\
\end{array}$} \\
\hline $\begin{array}{l}\text { State of } \\
\text { system }\end{array}$ & $\begin{array}{c}\text { Performance } \\
\text { capacity }\end{array}$ & $\begin{array}{c}\text { State of } \\
\text { subsystem } S_{1}\end{array}$ & $\begin{array}{l}\text { State of } \\
\text { Pipe \#3 }\end{array}$ \\
\hline \multirow{2}{*}{0} & \multirow{2}{*}{0.0 tons $/ \mathrm{min}$} & $0,1,2,3,4,5,6$ & 0 \\
\hline & & 0 & 1 \\
\hline 1 & 1.0 tons $/ \mathrm{min}$ & 1 & 1 \\
\hline 2 & 1.5 tons $/ \mathrm{min}$ & 2 & 1 \\
\hline 3 & 2.0 tons $/ \mathrm{min}$ & 3 & 1 \\
\hline 4 & 2.5 tons $/ \mathrm{min}$ & 4 & 1 \\
\hline 5 & 3.0 tons $/ \mathrm{min}$ & 5 & 1 \\
\hline 6 & 3.5 tons $/ \mathrm{min}$ & 6 & 1 \\
\hline
\end{tabular}

constant. The state probability $p_{l, i}(t)\left(i=0,1,2, \ldots, N_{C_{l}}\right)$ can be derived by solving the Kolmogorov differential equations:

$$
\frac{d p_{l, i}(t)}{d t}=\sum_{k=i+1}^{N_{C_{l}}} \lambda_{l,(k, i)} p_{l, k}(t)-p_{l, i}(t) \sum_{k=0}^{i-1} \lambda_{l,(i, k)},
$$

with the initial conditions that $p_{l, N_{C_{l}}}(0)=1$ and $p_{l, i}(0)=0$ ( $i \neq N_{C_{l}}$ ), that is, components are in the best state at the beginning of use. Note that, the parameters associated with the Markov models are unknown and need to be identified by the MSII [10].

(3) The structure function of subsystem $m\left(m=1,2, \ldots, M_{\text {sub }}\right)$ and the entire MSS, denoted by $\phi_{S_{m}}(\bullet)$ and $\phi_{S}(\bullet)$, respectively, are assumed to be deterministic and known. Therefore, the state of an MSS at any time $t$ can be completely determined by the combination of all the component states. For example, for the flow transmission system, the structure function $\phi_{S_{1}}(\cdot)$ is the sum of the performance capacities of Pipes \#1 and \#2. The structure function $\phi_{S}(\bullet)$ is the minimum of the performance capacities of the subsystem $S_{1}$ and Pipe \#3.

(4) Reliability of an MSS is defined as the probability that the system remains in acceptable states, and can be mathematically written as:

$$
R_{S}(t)=1-F_{S}(t)=1-\sum_{i \in \mathbf{D}} p_{S, i}(t),
$$

where $F_{S}(t)$ is the failure probability of the MSS, $p_{S, i}(t)$ is the probability of the system being in state $i$, and $\mathbf{D}$ is a set of unacceptable states. For instance, if the set of unacceptable states of the flow transmission system is defined as $\mathbf{D}=\{0,1\}$, i.e., the system flow rate is lower than 1.5 ton/min. The system reliability function can be, then, formulated as:

$$
\begin{aligned}
R_{S}(t) & =1-\sum_{i \in \mathbf{D}} p_{S, i}(t) \\
& =p_{3,1}(t) \times\left(p_{2,2}(t)+p_{2,1}(t)+p_{2,0}(t) \times p_{1,2}(t)\right)
\end{aligned}
$$

\section{B. Calculating the Reliability Bounds of MSSs By Fusing MSII}

For an MSS, MSII can be gathered from different experts in different forms and regarding distinct reliability measures. Examples of the MSII include: the interval values of components' state probabilities, interval values of the system's state probabilities, interval value of MTTF of the components/subsystems, and interval value of sojourn time at some particular states. In our previous work [10], a constrained optimization model was developed to evaluate the reliability bounds of MSSs, where the MSII is used to construct the constraints of the optimization model. For instance, if there are $e_{k}$ pieces of imprecise information provided by the $k$ th expert, the system reliability bounds can be found by:

$$
\begin{array}{ll}
{\left[R_{S L, k}(t), R_{S U, k}(t)\right]=\min / \max } & R_{S}(t) \\
\text { s.t. } & g_{i}\left(\lambda, t_{k}\right) \leq 0 \quad i=1,2, \ldots ., e_{k}
\end{array}
$$

where $g_{i}\left(\lambda, t_{k}\right)$ is the $i$ th normalized constraints provided by the $k$ th expert at time $t_{k} ; \boldsymbol{\lambda}$ is a vector of decision variables, which comprises of the transition intensities of all the components. By setting time $t$ in Eq. (4) at any time instant, the reliability bounds of the MSS over time can be assessed. Moreover, when there are $\sum_{k=1}^{K} e_{k}$ pieces of MSII elicited from $K$ experts, the reliability bounds of the MSS can be evaluated by the following constraint optimization model:

$$
\begin{aligned}
& {\left[R_{S L}(t), R_{S U}(t)\right]=\min / \max R_{S}(t)} \\
& \text { s.t. } \quad g_{i}\left(\lambda, t_{1}\right) \leq 0 \quad i=1,2, \ldots ., e_{1} \\
& g_{i}\left(\lambda, t_{2}\right) \leq 0 \quad i=1,2, \ldots, e_{2} . \\
& g_{i}\left(\lambda, t_{K}\right) \leq 0 \quad i=1,2, \ldots, e_{K}
\end{aligned}
$$

Note that, the reliability bound assessed by Eq. (5) should be no wider than that by Eq. (4), because there are more constraints in Eq. (5). To illustrate the use of Eqs. (4) and (5), here we consider a simple example of an MSS with only a three-state 
component where State 0 is the failure state, States 1 , and 2 are the working states. Suppose that the MSII is collected from two

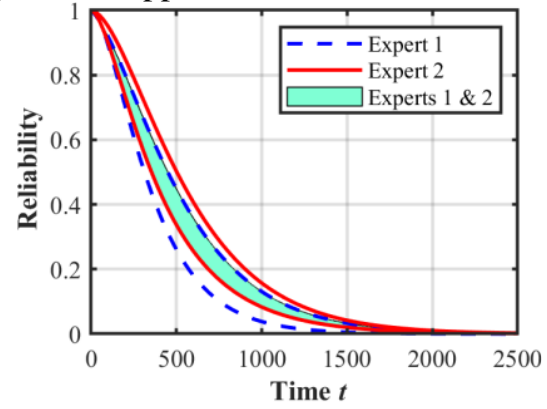

Fig. 2. Reliability bounds of the parallel-connected system by fusing the MSII.

experts:

Expert 1: The intervals of the state probabilities of the component at $t=300$ days are provided as $0.30 \leq p_{1,2}(300)$ $\leq 0.38 ; 0.22 \leq p_{1,1}(300) \leq 0.32 ; 0.30 \leq p_{1,0}(300) \leq 0.48$.

Expert 2: The interval of the MTTF of the component is $450 \leq M T T F_{1} \leq 600$ days.

The reliability bounds of the component can be obtained by considering the imprecise information from each expert separately:

$$
\begin{aligned}
& {\left[R_{S L, 1}(t), R_{S U, 1}(t)\right]=\min / \max R_{S}(t)=p_{1,2}(t)+p_{1,1}(t)} \\
& \text { s.t. }\left\{\begin{array}{l}
0.30 \leq p_{1,2}(300) \leq 0.38 \\
0.22 \leq p_{1,1}(300) \leq 0.32 \\
0.30 \leq p_{1,0}(300) \leq 0.48
\end{array}\right.
\end{aligned}
$$

and

$$
\begin{aligned}
& {\left[R_{S L, 2}(t), R_{S U, 2}(t)\right]=\min / \max \quad R_{S}(t)=p_{1,2}(t)+p_{1,1}(t)} \\
& \text { s.t. }\left\{\begin{array}{l}
450 \leq M T T F_{1} \leq 600 \\
M T T F_{1}=\int_{0}^{+\infty} R_{1}(t) d t=\int_{0}^{+\infty}\left(\sum_{i=1}^{2} p_{1, i}(t)\right) d t
\end{array} .\right.
\end{aligned}
$$

The MSII from the two experts can be fused to assess the reliability bound based on Eq. (5):

$$
\begin{aligned}
& {\left[R_{S L}(t), R_{S U}(t)\right]=\min / \max R_{S}(t)=p_{1,2}(t)+p_{1,1}(t)} \\
& \text { s.t. }\left\{\begin{array}{l}
0.30 \leq p_{1,2}(300) \leq 0.38 \\
0.22 \leq p_{1,1}(300) \leq 0.32 \\
0.30 \leq p_{1,0}(300) \leq 0.48 \\
450 \leq M T T F_{1} \leq 600
\end{array}\right.
\end{aligned}
$$

Figure 2 depicts the obtained reliability bounds. The results are achieved by running the optimization models in Eqs. (6)-(8) pointwisely at different values of $t$ from 0 to 2500 days. As shown in Fig. 2, fusing the two experts' MSII through Eq. (8), yields narrower reliability bounds. More importantly, as illustrated in Fig. 2, the reliability bounds from the individual expert MSII are not fully consistent, that is, conflicts still exist. To achieve a more rational consensus on the reliability estimates, the conflict should be properly quantified. Once the experts can see the conflicts between their judgements, they could exchange opinions and further calibrate their judgements. In the subsequent section, we present a two-dimensional conflict measure under the Dempster-Shafer theory to quantify and visualize the conflict among experts' MSII.

\section{A NEW CONFLICT MEASURE UNDER DST}

In this section, a new conflict measure under DST, named as two-dimensional conflict measure, is defined. The twodimensional conflict measure takes the conflict factor and the Jousselme distance as two complementary conflict measures. Before defining the new conflict measure, some basic definitions of DST are briefly introduced.

\section{A. Dempster-Shafer Theory}

DST is a generalized extension of probability theory by assigning probability masses to non-singleton sets [19],[20]. In general, let $\Omega$ be the frame of discernment (FoD) that contains all mutually exclusive propositions. A mass function, also called the BBA, is a mapping function $m: 2^{\Omega} \rightarrow[0,1]$ satisfying the normalization axiom:

$$
\sum_{A \subseteq \Omega} m(A)=1 .
$$

A mass function is said to be defined under the closed-world assumption if $m(\varnothing)=0$. If $m(A)>0$, the set $A$ is called a focal element. A simple mass is the mass function such that: $m(A)=s, m(\Omega)=1-s$ where $|A|=1$. The simple mass degenerates to a categorical mass if $s=1$, and a vacuous mass if $s=0$. Apart from the mass function, the DST employs an interval measure $[\operatorname{Bel}(A), P l(A)]$ to quantify the belief degree on set $A$. The lower bound, called the belief function, is defined as the total amount of the masses that support set $A$ :

$$
\operatorname{Bel}(A)=\sum_{B \subseteq A} m(B) .
$$

The upper bound, named as the plausibility function, is defined as the sum of all the masses of propositions that partially or totally agree with set $A$ :

$$
P l(A)=\sum_{B \cap A \neq \varnothing} m(B) .
$$

Given two mass functions $m_{1}$ and $m_{2}$ corresponding to two independent sources of evidence defined in the same FoD $\Omega$, the mass function $m_{1 \cap 2}=m_{1} \cap m_{2}$ resulting from the conjunctive rule of combination (CRC) is defined as:

$$
m_{1 \cap 2}(A)=\sum_{B \cap C=A, B, C \subseteq \Omega} m_{1}(B) m_{2}(C), \quad \forall A \subseteq \Omega .
$$

The CRC is proven to be commutative and associative, and it is often referred to as the unnormalized Dempster's rule. However, if the frame of discernment is assumed to be exhaustive, the mass $m(\varnothing)$ should be reallocated to other subsets, leading to the well-known Dempster's rule of combination $\oplus$ (DRC) (or orthogonal rule of combination):

$$
m_{1 \oplus 2}(A)=\left\{\begin{array}{lc}
\frac{m_{1 \cap 2}(A)}{1-k_{1 \oplus 2}}=\frac{m_{1 \cap 2}(A)}{1-m_{1 \oplus 2}(\varnothing)} & \text { if } A \subseteq \Omega \\
0 & \text { if } A=\varnothing
\end{array},\right.
$$

where

$$
k_{1 \oplus 2}=m_{1 \oplus 2}(\varnothing)=\sum_{B \cap C=\varnothing, B, C \subseteq \Omega} m_{1}(B) m_{2}(C)
$$

is the conflict factor between the two pieces of evidence. If there is no conflict between $m_{1}$ and $m_{2}$, the result of DRC is the same as that of CRC. Note that if $m_{1}\left(\right.$ or $\left.m_{2}\right)$ is a vacuous mass, the results of the DRC $m_{1 \oplus 2}$ is exactly the same as $m_{2}$ (or $m_{1}$ ). 
In this work, the conflict factor in the DRC, i.e., Eq. (14), plays an essential role in quantifying the conflict of MSII from a group of experts.

For an arbitrary mass function, its corresponding Pignistic probability function is defined as [29]:

$$
\operatorname{BetP}_{m}(A)=\sum_{B \subseteq \Omega} \frac{|B \cap A|}{|B|} \frac{m(B)}{1-m(\varnothing)} .
$$

This transformation is the so-called Pignistic transformation (PT). The Pignisctic probability function $\operatorname{Bet}_{m}(A)$ is also called the betting commitment to set $A$.

Apart from the conflict factor, there are many other conflict measures developed from various perspectives in the literature. The cosine measure is a well-known inner product measure of similarity in the DST, which is defined as:

$$
\cos \left(m_{1}, m_{2}\right)=\frac{m_{1} \bullet m_{2}}{\left\|m_{1}\right\| \times\left\|m_{2}\right\|}
$$

where $m_{1} \cdot m_{2}$ is the inner product of two vectors $m_{1}$ and $m_{2}$, $\|\cdot\|$ is the length of the vector. Thereby, the conflict between $m_{1}$ and $m_{2}$ is $1-\cos \left(m_{1}, m_{2}\right)$.

Moreover, the supremum norm of the difference of betting commitment, denoted as $\operatorname{diffBetP}_{m_{j}}^{m_{i}}$, is also a well-known distance measure to measure the conflict in the DST. $\operatorname{diffBetP} m_{m_{2}}^{m_{1}}$ of two pieces of BBAs $m_{1}$ and $m_{2}$ can be written as:

$$
\begin{aligned}
\operatorname{diffBetP} m_{m_{2}}^{m_{1}} & =\left\|\operatorname{BetP}_{m_{1}}(A)-\operatorname{BetP}_{m_{2}}(A)\right\|_{\infty} \\
& =\max _{A \subseteq \Omega}\left(\left|\operatorname{Bet}_{m_{1}}(A)-\operatorname{BetP}_{m_{2}}(A)\right|\right)
\end{aligned}
$$

where $\operatorname{Bet}_{m_{1}}(A)$ and $\operatorname{Bet}_{m_{2}}(A)$ are the betting commitments of element $A(A \subseteq \Omega)$ induced by $m_{1}$ and $m_{2}$ respectively.

\section{B. The Two-Dimensional Conflict Measure}

In DST, the conflict factor is the most intensively used measure to quantify the conflict among evidences. Without loss of generality, let the FoD of the studied system be $\Omega=\{W, F\}$ where $W$ and $F$ represent the working and failed states of the system, respectively. Then, the power set is $2^{\Omega}=\{\varnothing, W, F,\{W, F\}\}$. Let $\mathbf{m}_{i}(t), \mathbf{m}_{j}(t)$ be two BBAs assigned to the power set $2^{\Omega}$ from the $i$ th and $j$ th experts at time $t$, respectively. The conflict between these two pieces of BBAs can be quantified by:

$$
k_{i \oplus j}(t)=m_{i \oplus j}^{\varnothing}(t)=\sum_{A \cap B=\varnothing, A, B \subseteq \Omega} m_{i}^{A}(t) m_{j}^{B}(t),
$$

where $m_{i}^{A}(t)$ and $m_{j}^{B}(t)$ stand for the mass functions of $\mathbf{m}_{i}(t)$ , $\mathbf{m}_{j}(t)$, respectively. In reliability engineering, the mass functions can be obtained by converting the interval-valued reliability estimates from experts into mass beliefs (see Section IV.A for details). The range of $k_{i \oplus j}(t)$ is $[0,1]$, where $k_{i \oplus j}(t)=0$ means no conflict exists between the two experts, while $k_{i \oplus j}(t)=1$ represents the two experts are totally contradicting. Sometimes, the experts may be interested in the conflict of their BBAs within a specific time interval. Based on Eq. (18), the average conflict factor for the $i$ th and $j$ th experts within an interval $T_{\text {int }}=\left[T_{L}, T_{U}\right]$ can be calculated as:

$$
\begin{aligned}
k_{i \oplus j}=m_{i \oplus j}^{\varnothing} & =\frac{1}{T_{U}-T_{L}} \int_{t \in T_{\text {int }}} k_{i \oplus j}(t) d t \\
& =\frac{1}{T_{U}-T_{L}} \int_{t \in T_{\text {int }}} \sum_{A \cap B=\varnothing, A, B \subseteq \Omega} m_{i}^{A}(t) m_{j}^{B}(t) d t
\end{aligned}
$$

The average conflict factor $k_{i \oplus j}$ has the following properties.

Property 1 (Bounded): The range of the average conflict factor is $0 \leq k_{i \oplus j} \leq 1$. Between the two extremes, a greater value of $k_{i \oplus j}$ indicates higher degree of conflicts. $k_{i \oplus j}=1$ if and only if the two experts' BBAs are totally conflict. In other words, the studied system is completely working according to one expert while totally failed according to the other. $k_{i \oplus j}=0$ holds if and only if the two experts' BBAs are two simple BBAs with the same focal elements.

Property 2. (Commutative): The average conflict factor is commutative, i.e., $k_{i \oplus j}=k_{j \oplus i}$.

Property 3. (Imprecision Monotonicity): The average conflict factor is nonincreasing when the imprecision of anyone of the experts' information increases, i.e., $k_{i \oplus j}<k_{i^{\prime} \oplus j}$ with $\mathbf{m}_{i^{\prime}}(t)$ is a piece of more precise information than $\mathbf{m}_{i}(t)$, i.e., $\mathbf{m}_{i^{\prime}}(t)$ has less masses assigned to set $\{W, F\}$ than $\mathbf{m}_{i}(t)$.

Property 4. (Non-Conflict to Ignorance): The average conflict factor is not in conflict with ignorance, i.e., the average conflict factor of any BBAs with respect to the ignorance is zero. It indicates that if one expert tells nothing about the reliability of a system, it should not be conflicting with any information from the other experts.

Detailed proofs of Properties 1-4 can be found in Appendix A. these properties match well with our intuitive anticipation of a conflict measure. However, the conflict factor has been criticized for failing to quantify the dissimilarity between two identical BBAs [18]. Put it in another way, if two BBAs are exactly the same, the conflict factor may not be equal to 0 , which is a somewhat counterintuitive result. A plethora of researches have pointed out that using the conflict factor as the unique measure of conflict may be misleading while integrating every aspect of conflict into a single measurement is challenging [18],[22]. Therefore, we propose to complement the conflict factor with other conflict measures. In DST, the distance is another effective measure to quantify the conflict between BBAs. If two BBAs are conflicting, they should be far from each other. Many well-known distance measures, including the Jousselme distance [23], the supremum norm of the difference of betting commitment [18], and the TBM pairwise dissimilarity measure [30], have been proposed and implemented on a diversity of applications, such as optimization in classification algorithms, parameter estimation of combination rules, etc. However, it is noteworthy that none of the distance measures prevails on all the conflict quantification problems in DST. The choice of an appropriate distance measure should be application-specific. Among the distance measures in DST, the Jousselme distance is one of the most widely-used measure due to its satisfactory properties on measuring the dissimilarity between BBAs [25]. In this work, the Jousselme distance serves as a complementary measure of 
the conflict factor. For two BBAs $\mathbf{m}_{i}(t)$ and $\mathbf{m}_{j}(t)$ from the $i$ th and $j$ th experts at time $t$, the Jousselme distance is given by:

$$
J D\left(\mathbf{m}_{i}(t), \mathbf{m}_{j}(t)\right)=\sqrt{\frac{1}{2}\left(\mathbf{m}_{i}(t)-\mathbf{m}_{j}(t)\right) \mathbf{J a c}\left(\mathbf{m}_{i}(t)-\mathbf{m}_{j}(t)\right)^{T}},
$$

where $\left(\mathbf{m}_{i}(t)-\mathbf{m}_{j}(t)\right)^{T}$ stands for the transpose of the vector $\mathbf{m}_{i}(t)-\mathbf{m}_{j}(t)$. Jac is a $2^{\Omega} \times 2^{\Omega}$-dimensional matrix whose elements are calculated by the Jaccard index, that is:

$$
\operatorname{Jac}(A, B)=\frac{|A \cap B|}{|A \cup B|}, \quad \forall A, B \subseteq \Omega .
$$

The range of $J D\left(\mathbf{m}_{i}(t), \mathbf{m}_{j}(t)\right)$ is also $[0,1]$, where $J D\left(\mathbf{m}_{i}(t), \mathbf{m}_{j}(t)\right)=0$ holds if and only if the two BBAs are identical. $J D\left(\mathbf{m}_{i}(t), \mathbf{m}_{j}(t)\right)=1$ represents the two experts are totally contradicting. If the experts are interested in the distance of their BBAs within a specific time interval, the average Jousselme distance of the $i$ th and $j$ th experts' BBAs within an interval $T_{\text {int }}=\left[T_{L}, T_{U}\right]$ can be calculated as:

$$
J D\left(\mathbf{m}_{i}, \mathbf{m}_{j}\right)=\frac{1}{T_{U}-T_{L}} \int_{t \in T_{\mathrm{int}}} J D\left(\mathbf{m}_{i}(t), \mathbf{m}_{j}(t)\right) d t .
$$

It can be proved that the proposed average Jousselme distance of BBAs has the following properties (See Appendix B for the proofs).

Property 5. (Bounded): The range of the average distance is $0 \leq J D\left(\mathbf{m}_{i}, \mathbf{m}_{j}\right) \leq 1$. Between the two extremes, a greater value of $J D\left(\mathbf{m}_{i}, \mathbf{m}_{j}\right)$ indicates higher degree of conflict.

Property 6. (Extreme Consistent Value): $J D\left(\mathbf{m}_{i}, \mathbf{m}_{j}\right)=0$ holds if and only if the two BBAs are exactly the same; $J D\left(\mathbf{m}_{i}, \mathbf{m}_{j}\right)=1$ holds if and only if the system is completely working believed by one expert while totally failed believed by the other.

Property 7. (Commutative): $J D\left(\mathbf{m}_{i}, \mathbf{m}_{j}\right)=J D\left(\mathbf{m}_{j}, \mathbf{m}_{i}\right)$.

Property 8. (Conflict to Ignorance): $J D\left(\mathbf{m}_{i}, \mathbf{m}_{j}\right)$ is sensitive to ignorance. To be more specific, let $\mathbf{m}_{j}=(0,0,0,1)$ be a vacuous BBA, we have

$$
J D\left(\mathbf{m}_{i},(0,0,0,1)\right)=\frac{1}{T_{U}-T_{L}} \int_{t \in T_{\text {int }}} \sqrt{\frac{1}{2}\left(\left\|\mathbf{m}_{i}\right\|^{2}-m_{i}^{\Omega}\right)} d t \geq 0,
$$

where $\left\|\mathbf{m}_{i}\right\|^{2}$ is the square norm of $\mathbf{m}_{i}$, the equality holds if and only if $\mathbf{m}_{i}$ is also a vacuous BBA.

As concluded from Properties 1, 2, 5, and 6, both $k_{i \oplus j}$ and $J D\left(\mathbf{m}_{i}, \mathbf{m}_{j}\right)$ reach the maximum when the two BBAs are totally contradictory. However, $k_{i \oplus j}$ and $J D\left(\mathbf{m}_{i}, \mathbf{m}_{j}\right)$ reach the minimum under different scenarios: $k_{i \oplus j}$ reaches the minimum when two BBAs are identical simple BBAs, whereas $J D\left(\mathbf{m}_{i}, \mathbf{m}_{j}\right)$ reaches its minimum when the two BBAs are identical. Property 8 of the average Jousselme distance is dissatisfied as a conflict measure, because when one expert tells nothing about the system reliability, it should not be conflicting with any other expert judgements. Both the $k_{i \oplus j}$ and $J D\left(\mathbf{m}_{i}, \mathbf{m}_{j}\right)$ have their shortcomings in measuring the conflict: $k_{i \oplus j}$ is prone to measure the conflict to ignorance while $J D\left(\mathbf{m}_{i}, \mathbf{m}_{j}\right)$ is prone to measure the conflict of two identical BBAs. However, $k_{i \oplus j}$ and $J D\left(\mathbf{m}_{i}, \mathbf{m}_{j}\right)$ can complement the shortcomings of each other and provide a comprehensive conflict quantification. Hence, a two-dimensional conflict measure, which integrates the average conflict factor $k_{i \oplus j}$ and the average Jousselme distance $J D\left(\mathbf{m}_{i}, \mathbf{m}_{j}\right)$, is proposed in this work to quantify the conflict among experts' BBAs.

Definition 1: Let $\mathbf{m}_{i}$ and $\mathbf{m}_{j}$ be two BBAs. Let $\operatorname{TDCM}\left(\mathbf{m}_{i}, \mathbf{m}_{j}\right)=<k_{i \oplus j}, J D\left(\mathbf{m}_{i}, \mathbf{m}_{j}\right)>$ be a two-dimensional conflict measure where $k_{i \oplus j}$ is the uncommitted masses when combining $\mathbf{m}_{i}$ and $\mathbf{m}_{j}$ via DRC and $J D\left(\mathbf{m}_{i}, \mathbf{m}_{j}\right)$ is the distance between $\mathbf{m}_{i}$ and $\mathbf{m}_{j} . \mathbf{m}_{i}$ and $\mathbf{m}_{j}$ are defined as in conflict if and only if both $k_{i \oplus j}>\varepsilon$ and $J D\left(\mathbf{m}_{i}, \mathbf{m}_{j}\right)>\varepsilon$ hold, where $\varepsilon$ is a pre-defined threshold of conflict tolerance.

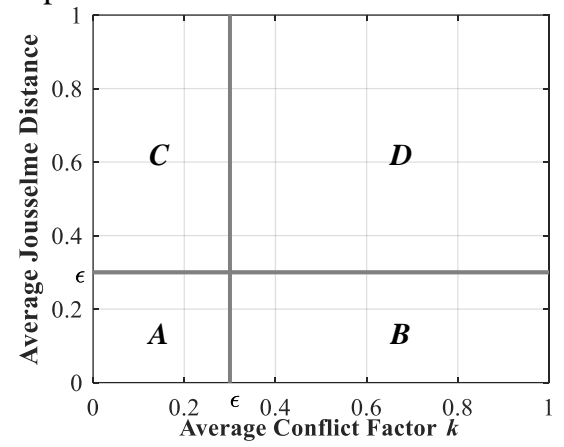

Fig. 3. Conflict quantification and visualization by the two-dimensional conflict measure.

The proposed two-dimensional conflict measure $\operatorname{TDCM}\left(\mathbf{m}_{i}, \mathbf{m}_{j}\right)=<k_{i \oplus j}, J D\left(\mathbf{m}_{i}, \mathbf{m}_{j}\right)>$ satisfies the following two lemmas.

Lemma 1: $\operatorname{TDCM}\left(\mathbf{m}_{i}, \mathbf{m}_{j}\right)=<1,1>$ if and only if $\left(\cup A_{i}\right) \cap\left(\cup B_{j}\right)=\varnothing$, where $A_{i}$ and $B_{j}$ are the focal elements of the BBAs of $\mathbf{m}_{i}$ and $\mathbf{m}_{j} .\left(\cup A_{i}\right) \cap\left(\cup B_{j}\right)=\varnothing$ means the focal elements of $\mathbf{m}_{i}$ and $\mathbf{m}_{j}$ are totally different. Therefore, the FoD cannot be a focal element for both $\mathbf{m}_{i}$ and $\mathbf{m}_{j}$.

Lemma 2: $\operatorname{TDCM}\left(\mathbf{m}_{i}, \mathbf{m}_{j}\right)=<0,0>$ if and only if both $\mathbf{m}_{i}$ and $\mathbf{m}_{j}$ are two identical simple BBAs. This can be readily concluded from that: $k_{i \oplus j}=0$ if and only if $\mathbf{m}_{i}$ and $\mathbf{m}_{j}$ are two simple BBAs with the same focal elements, while $J D\left(\mathbf{m}_{i}, \mathbf{m}_{j}\right)=0$ means the two BBAs are identical.

The above two lemmas of $\operatorname{TDCM}\left(\mathbf{m}_{i}, \mathbf{m}_{j}\right)$ can be readily proofed by the properties of the average conflict factor and average Jousselme distance. As concluded from Lemma 1, $\operatorname{TDCM}\left(\mathbf{m}_{i}, \mathbf{m}_{j}\right)$ reaches its maximum value of $\left.<1,1\right\rangle$ when the system is believed to be working by one expert while believed to be failed by another expert. As concluded from Lemma 2, $\operatorname{TDCM}\left(\mathbf{m}_{i}, \mathbf{m}_{j}\right)$ reaches its minimum value of $<0,0>$ when both experts assign the same masses to one of the singletons between $\{W\}$ and $\{F\}$ while the other masses remain unassigned. In practice, the two-dimensional conflict measure $\operatorname{TDCM}\left(\mathbf{m}_{i}, \mathbf{m}_{j}\right)$ might lies in different regions of the plane, resulting in four typical scenarios with different degrees of conflicts, as shown in Fig. 3.

Case A: When both the average conflict factor $k_{i \oplus j}$ and the average Jousselme distance $J D\left(\mathbf{m}_{i}, \mathbf{m}_{j}\right)$ have low values, i.e., $k_{i \oplus j} \leq \varepsilon$ and $J D\left(\mathbf{m}_{i}, \mathbf{m}_{j}\right) \leq \varepsilon$, the two-dimensional conflict measure $<k_{i \oplus j}, J D\left(\mathbf{m}_{i}, \mathbf{m}_{j}\right)>$ indicates that the two BBAs are not in conflict. It means these two experts has a high degree of consensus.

Case B: When the average conflict factor $k_{i \oplus j}$ has a relatively high value while the average Jousselme distance $J D\left(\mathbf{m}_{i}, \mathbf{m}_{j}\right)$ has a low value, i.e., $k_{i \oplus j}>\varepsilon$ and 
$J D\left(\mathbf{m}_{i}, \mathbf{m}_{j}\right) \leq \varepsilon$, the two-dimensional conflict measure $\left.<k_{i \oplus j}, J D\left(\mathbf{m}_{i}, \mathbf{m}_{j}\right)\right\rangle$ indicates that the two BBAs are nearly distributed evenly on the singletons, i.e., $\mathbf{m}_{i} \approx \mathbf{m}_{j} \approx(0,0.5,0.5,0)$.

Case $C$ : When the average conflict factor $k_{i \oplus j}$ has a low value while the average Jousselme distance $J D\left(\mathbf{m}_{i}, \mathbf{m}_{j}\right)$ has a relatively high value, i.e., $k_{i \oplus j} \leq \varepsilon$ and $J D\left(\mathbf{m}_{i}, \mathbf{m}_{j}\right)>\varepsilon$, the two-dimensional conflict measure $\left\langle k_{i \oplus j}, J D\left(\mathbf{m}_{i}, \mathbf{m}_{j}\right)\right\rangle$ indicates that one of the experts provides very limited information to the system reliability estimate, i.e., the mass assigned to $m_{i}^{\{W, F\}}$ or $m_{j}^{\{W, F\}}$ is very high.

Case D: When both the average conflict factor $k_{i \oplus j}$ and the average Jousselme distance $J D\left(\mathbf{m}_{i}, \mathbf{m}_{j}\right)$ have high values, the two-dimensional conflict measure $\left\langle k_{i \oplus j}, J D\left(\mathbf{m}_{i}, \mathbf{m}_{j}\right)\right\rangle$ indicates that the two BBAs are in conflict.

In Fig. 3, the two regions, denoted by $D$ and $A$, corresponds to the situation that the two experts are in and not in conflict, respectively. The region $B$ shows that even the average conflict factor $k_{i \oplus j}$ is high, the two experts are not contradictory to each other. It is indeed the region that can be misled when only using the average conflict factor. The region $C$ reflects that the conflict of two experts is small but the reason is that one of the experts provides very few useful information.

In the literature, Liu [18] introduced to use the supremum norm of the difference of betting commitment $\operatorname{diffBetP} \mathbf{m}_{\mathbf{m}_{i}}^{\mathbf{m}_{i}}$, to compensate for the counterintuitive result of the conflict factor. However, we note that $\operatorname{diffBet} \mathrm{P}_{\mathbf{m}_{j}}^{\mathbf{m}_{i}}$ cannot be used in this work to compensate for the conflict factor as it is unable to accurately quantify the distance of BBAs in some particular cases.

Let us consider two reliability bounds $[0.6,0.7]$ and $[0.5$, $0.8]$ induced from two experts' MSII, the corresponding BBAs and betting commitments are given in Table IV. $\operatorname{diffBetP} \mathrm{P}_{\mathbf{m}_{2}}^{\mathbf{m}_{1}}$ can be computed as:

$$
\begin{aligned}
\operatorname{diffBetP} \mathbf{m}_{\mathbf{m}_{2}}^{\mathbf{m}_{1}} & =\max _{A \subseteq \Omega}\left(\left|\operatorname{Bet}_{\mathbf{m}_{1}}(A)-\operatorname{BetP}_{\mathbf{m}_{2}}(A)\right|\right) \\
& =\max (|0.65-0.65|,|0.35-0.35|) . \\
& =0
\end{aligned}
$$

The Jousselme distance between $\mathbf{m}_{1}$ and $\mathbf{m}_{2}$ can be calculated by:

$$
\begin{aligned}
& J D\left(\mathbf{m}_{1}, \mathbf{m}_{2}\right) \\
& =\sqrt{0.5 \times(0,0.1,0.1,-0.2)\left[\begin{array}{cccc}
1 & 0 & 0 & 0 \\
0 & 1 & 0 & 0.5 \\
0 & 0 & 1 & 0.5 \\
0 & 0.5 & 0.5 & 1
\end{array}\right](0,0.1,0.1,-0.2)^{T} .}
\end{aligned}
$$$$
=0.1
$$

It is obvious that $\mathbf{m}_{1}$ and $\mathbf{m}_{2}$ are not identical and conflict should exist. However, $\operatorname{diffBetP_{\mathbf {m}_{2}}^{\mathbf {m}_{1}}}$ tells us these two BBAs are consistent. Therefore, $\operatorname{diffBet} \mathrm{P}_{\mathbf{m}_{2}}^{\mathbf{m}_{1}}$ is incapable of handling such case of BBAs.

TABLE IV THE INDUCED BBA AND BETTING COMMITMENTS OF TWO EXEMPLIFIED RELIABILITY BOUNDS

\begin{tabular}{ccc}
\hline \hline Reliability bounds & BBAs & Betting commitments \\
\hline$\left[R_{S L, 1}, R_{S U, 1}\right]=[0.6,0.7]$ & $\mathbf{m}_{1}=(0,0.6,0.3,0.1)$ & $\operatorname{Bet} P_{\mathbf{m}_{1}}=(0,0.65,0.35,0)$ \\
{$\left[R_{S L, 2}, R_{S U, 2}\right]=[0.5,0.8]$} & $\mathbf{m}_{2}=(0,0.5,0.2,0.3)$ & $\operatorname{Bet} P_{\mathbf{m}_{2}}=(0,0.65,0.35,0)$ \\
\hline \hline
\end{tabular}

Another interesting question is how large should $\varepsilon$ be of the two-dimensional conflict measure. In the literature, Smets [31] used the relation $\varepsilon=1-(1-c)^{K}$ to decide the tolerable conflict under the open-world assumption, where $c$ is the maximum of the mass function of the focal element $\varnothing$ in the $K$ number of evidences. However, it is noted that $m(\varnothing)=0$ always hold in this article as shown in Eq. (23), thereby Smets' method cannot be applied to our specific problem. Under the close-world assumption, it is generally acknowledged that there does not

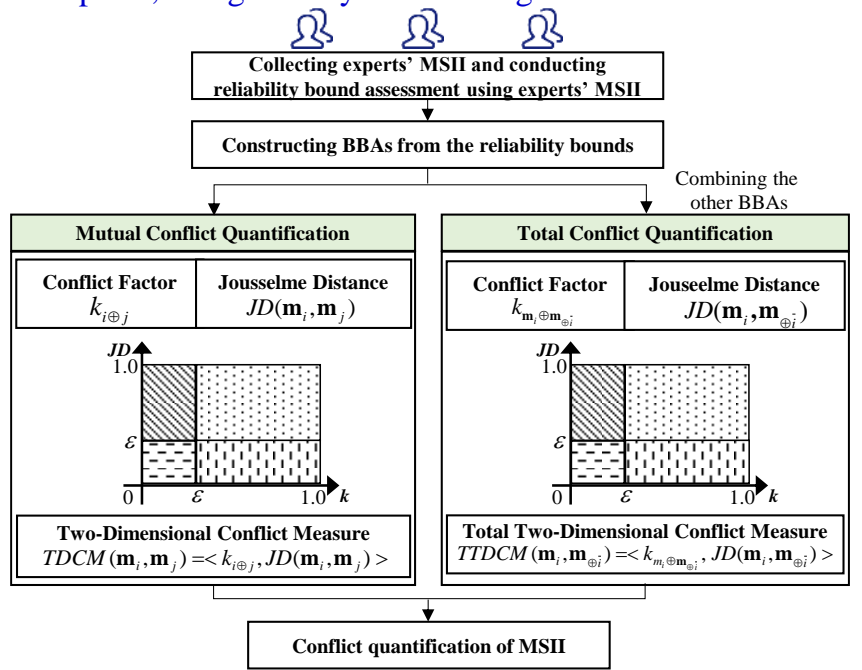

Fig. 4. The framework of the conflict quantification of experts' MSII.

exist an "absolute meaningful threshold" of conflict tolerance for any pairs of BBAs, the choice of $\varepsilon$ is largely subjective and application oriented [18]. In the data fusion field by the DRC, there exists an important rule to determine whether or not the DRC can be applied for two pieces of BBAs based on the conflict factor [18]. The rule is illustrated by the following three cases:

Case 1: $k_{i \oplus j}>\varepsilon_{2}$ where $\varepsilon_{2}$ is set to 0.8 , this case indicates that the DRC cannot be used as it may generate counterintuitive results.

Case 2: $k_{i \oplus j}<\varepsilon_{1}$ where $\varepsilon_{1}$ is set to 0.3 , the DRC is perfectly applicable.

Case 3: $\varepsilon_{1} \leq k_{i \oplus j} \leq \varepsilon_{2}$. It advises that DRC should be used cautiously in order not to eliminate the true proposition during combination.

In this article, only a small amount of conflict between two experts' MSII is tolerated, $\varepsilon=0.3$ is, therefore, selected as our threshold of conflict tolerance. $\varepsilon$ can also be selected between 0.3 and 0.8 , which means more conflict is to be tolerated of any two pieces of BBAs.

\section{QuANTIFYING CONFLICT BASED ON THE Two- DIMENSIONAL MEASURE}

In this section, the two-dimensional conflict measure is used to quantify and visualize the conflict among experts' MSII. Figure 4 illustrates the framework of the conflict quantification of experts' MSII by the two-dimensional conflict measure. First, the reliability bound of the studied system is evaluated by using the collected experts' MSII, as given in Eq. (4). Second, the reliability bounds induced by the MSII of each expert are converted into the corresponding basic belief assignment 
(BBA) under the DST (See details in Section IV.A). Thereby, the conflicts among all the experts' BBAs are measured from two aspects, i.e., the mutual conflict quantification and total conflict quantification. The mutual conflict quantification using the two-dimensional conflict measure to quantify the conflict between any two experts' BBAs (See details in Section IV.B), whereas the total conflict quantification using the total twodimensional conflict measure to quantify the conflict of each expert's BBAs with respect to the combined BBAs of the other experts via Dempster's rule of combination (DRC) (See details in Section IV.B).

\section{A. Constructing BBA From Reliability Bounds}

In this section, we formulate a method of constructing BBAs from reliability bounds given by experts. Based on the developed constrained optimization model in Eq. (4), the reliability bound of the system given by a single expert can be obtained by fusing the different pieces of MSII from the same expert. Let $\left[R_{S L, k}(t), R_{S U, k}(t)\right]$ represent the reliability bound from the $k$ th expert. Under the DST framework, the interval value of a probability measure is interpreted as the minimum and maximum belief that the expert assigned to the event that the system is working. Therefore, the lower and upper bounds of system reliability can be considered as the belief and plausibility measures of the proposition "The $k$ th expert believes that the system is working at time $t$ ", namely, $\left[R_{S L, k}(t), R_{S U, k}(t)\right]=\left[B e l_{S, k}^{W}(t), P l_{S, k}^{W}(t)\right]$. The quantity of $P l_{S, k}^{W}(t)-B e l_{S, k}^{W}(t)$ represents the ignorance of the $k$ th expert on this proposition. Thereby, the BBA associated with the $k$ th expert can be formulated by:

$$
\begin{aligned}
\mathbf{m}_{k}(t) & =\left(m_{k}^{\varnothing}(t), m_{k}^{W}(t), m_{k}^{F}(t), m_{k}^{\{W, F\}}(t)\right) \\
& =\left(0, R_{S L, k}(t), 1-R_{S U, k}(t), R_{S U, k}(t)-R_{S L, k}(t)\right)
\end{aligned}
$$

where $m_{k}^{\varnothing}(t)=0$ stands for the closed-world assumption of the mass function; $\quad m_{k}^{W}(t)=R_{S L, k}(t) \quad$ and $\quad m_{k}^{F}(t)=1-R_{S U, k}(t)$ represent the mass functions of system working and failure at time instant $t$ believed by the $k$ th expert, respectively; $m_{k}^{\{W, F\}}(t)=R_{S U, k}(t)-R_{S L, k}(t)$ is the mass function of system being in either working or failed states at time instant $t$ believed by the $k$ th expert. If the MSII is elicited from $K$ experts, therefore, $K$ pieces of evidences of the system reliability estimate can be obtained, represented as $\mathbf{m}_{1}(t), \mathbf{m}_{2}(t), \ldots, \mathbf{m}_{K}(t)$ . Subsequently, the proposed two-dimensional conflict measure is to be utilized to quantify the inconsistency among the BBAs of the $K$ experts.

\section{B. Mutual Conflict and Total Conflict Quantification}

As stated previously, we quantify the conflicts among experts' BBAs from the perspective of mutual conflict and total conflict. The mutual conflict can be done by applying the twodimensional conflict measure on each pair of the experts' BBAs. Let $\mathbf{m}_{i}$ and $\mathbf{m}_{j}$ be two BBAs from the $i$ th and $j$ th experts calculated by Eq. (23), the two-dimensional conflict measure $\operatorname{TDCM}\left(\mathbf{m}_{i}, \mathbf{m}_{j}\right)$ of these two experts can be calculated by Eqs. (19) and (22). The result of the twodimensional conflict measure $\operatorname{TDCM}\left(\mathbf{m}_{i}, \mathbf{m}_{j}\right)$ can be depicted in Fig. 4, which illustrates the conflicts between $\mathbf{m}_{i}$ and $\mathbf{m}_{j}$.
Sometimes, we are interested in the total conflict of one expert's BBA with respect to all the other experts' BBAs. The total conflict measure can be defined in a similar way as the mutual conflict measure. Let $\mathbf{m}_{\oplus \bar{i}}$ be the combined BBAs via DRC without the $i$ th experts' BBA, that is, $\mathbf{m}_{\oplus \bar{i}}=\oplus_{\oplus i} \mathbf{m}_{j}$, the total conflict between the $i$ th expert's BBA $\mathbf{m}_{i}$ and ${ }^{j i} \mathbf{m}_{\oplus \bar{i}}$ can be defined as:

$$
\operatorname{TTDCM}\left(\mathbf{m}_{i}, \mathbf{m}_{\oplus \bar{i}}\right)=<k_{\mathbf{m}_{i} \oplus \mathbf{m}_{\oplus \bar{i}}}, J D\left(\mathbf{m}_{i}, \mathbf{m}_{\oplus \bar{i}}\right)>,
$$

where the total average conflict factor $k_{\mathbf{m}_{i} \oplus \mathbf{m}_{\oplus \bar{i}}}$ can be calculated by:

$$
\begin{aligned}
k_{\mathbf{m}_{i} \oplus \mathbf{m}_{\oplus \bar{i}}} & =\frac{1}{T_{U}-T_{L}} \int_{t \in T_{\text {int }}} k_{\mathbf{m}_{i} \oplus \mathbf{m}_{\oplus \bar{i}}}(t) d t \\
& =\frac{1}{T_{U}-T_{L}} \int_{t \in T_{\mathrm{int}}} \sum_{A \cap B=\varnothing, A, B \subseteq \Omega} m_{i}^{A}(t) m_{\oplus \bar{i}}^{B}(t) d t
\end{aligned}
$$

and the total average Jousselme distance $J D\left(\mathbf{m}_{i}, \mathbf{m}_{\oplus \bar{i}}\right)$ can be calculated by:

$$
\begin{aligned}
& J D\left(\mathbf{m}_{i}, \mathbf{m}_{\oplus \bar{i}}\right)=\frac{1}{T_{U}-T_{L}} \int_{t \in T_{\mathrm{int}}} J D\left(\mathbf{m}_{i}(t), \mathbf{m}_{\oplus \bar{i}}(t)\right) d t \\
& =\frac{1}{T_{U}-T_{L}} \int_{t \in T_{\mathrm{int}}} \sqrt{\frac{1}{2}\left(\mathbf{m}_{i}(t)-\mathbf{m}_{\oplus \bar{i}}(t)\right)^{T} \mathbf{J a c}\left(\mathbf{m}_{i}(t)-\mathbf{m}_{\oplus \bar{i}}(t)\right)} d t
\end{aligned}
$$

where the Jaccard index matrix Jac is the same as that of in Eq. (21) as the FoD of the combined BBA $\mathbf{m}_{\oplus \bar{i}}$ is unchanged. Note that the total average conflict factor $k_{\mathbf{m}_{i} \oplus \mathbf{m}_{\oplus \bar{i}}}$ and the total average Jousselme distance $J D\left(\mathbf{m}_{i}, \mathbf{m}_{\oplus \bar{i}}\right)$ also satisfy the foregoing properties because $\mathbf{m}_{\oplus \bar{i}}$ is still a normalized BBA after the DRC.

\section{INFORMATIVENESS OF MSII}

In reality, experts are not only interested in the conflict among their MSII, but also how much information does each expert's MSII provide. The latter is measured by introducing informativeness in this paper. More specifically, a Bhattacharya distance-based informativeness is proposed to measure the informativeness of the $i$ th expert's BBA. The Bhattacharyya distance is a well-known similarity measure for two probability distributions that has been widely used in many fields, such as feature extraction [33], image processing [34], and speaker recognition[35]. In this work, the Bhattacharyya distance is used to measure the similarity of the PDFs of the upper and lower system lifetime distributions. Let $q_{R_{S U, k}(t)}, q_{R_{S L, k}(t)}$ be the PDFs of the upper and lower system lifetime distributions by fusing the MSII of the $k$ th expert, respectively, the Bhattacharyya distance $\delta_{B}\left(q_{R_{S U, k}(t)}, q_{R_{S L, k}(t)}\right)$ is mathematically defined as:

$$
\delta_{B}\left(q_{R_{S U, k}(t)}, q_{R_{S L, k}(t)}\right)=-\ln \left(\int_{t=0}^{+\infty} \sqrt{q_{R_{S U, k}(t)} \times q_{R_{S L, k}(t)}} d t\right),
$$

where $q_{R_{S U, k}(t)}=-d R_{S U, k}(t) / d t$ and $q_{R_{S L, k}(t)}=-d R_{S L, k}(t) / d t$. The Bhattacharyya distance ranges from 0 to $+\infty$. The smaller the Bhattacharyya distance is, the more imprecise of the reliability bound $\left[R_{S L, k}(t), R_{S U, k}(t)\right]$.

The basic procedures of the Bhattacharyya distance-based informativeness are illustrated in Fig. 5. It mainly contains the following three steps. 
Step 1: Evaluate the system reliability bounds $\left[R_{S L}(t), R_{S U}(t)\right]$ by fusing all experts' MSII using Eq. (5), and then, calculating the PDFs of the upper and lower system lifetime distributions, denoted as $q_{R_{S U}(t)}$ and $q_{R_{S L}(t)}$ : $q_{R_{S U}(t)}=-d R_{S U}(t) / d t$ and $q_{R_{S L}(t)}=-d R_{S L}(t) / d t$.

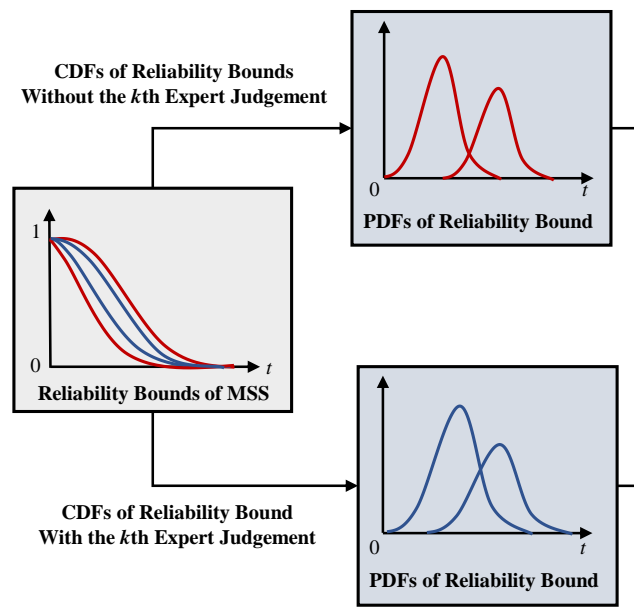

Fig. 5. The basic procedures of the Bhattacharyya distance-based informativeness.

Step 3: Calculate the Bhattacharyya distances of the resulting pairs of PDFs from Steps 1 and 2 [26]:

$$
\delta_{B}\left(q_{R_{S U}(t)}, q_{R_{S L}(t)}\right)=-\ln \left(\int_{t=0}^{+\infty} \sqrt{q_{R_{S U}(t)} \times q_{R_{S L}(t)}} d t\right),
$$

and

$$
\delta_{B}\left(q_{R_{S U, \bar{k}}(t)}, q_{R_{S L, \bar{k}}(t)}\right)=-\ln \left(\int_{t=0}^{+\infty} \sqrt{q_{R_{S U, \bar{k}}(t)} \times q_{R_{S L, \bar{k}}(t)}} d t\right) .
$$

Then, the Bhattacharya distance-based informativeness of the $k$ th expert's MSII can be defined as:

$$
\begin{aligned}
I_{B}\left(\mathbf{m}_{k}\right) & =\delta_{B}\left(q_{R_{S U, \bar{k}}(t)}, q_{R_{S L, \bar{k}}(t)}\right)-\delta_{B}\left(q_{R_{S U}(t)}, q_{R_{S L}(t)}\right) \\
& =\ln \left(\frac{\int_{t=0}^{+\infty} \sqrt{q_{R_{S U}(t)} \times q_{R_{S L}(t)}} d t}{\int_{t=0}^{+\infty} \sqrt{q_{R_{S U, \bar{k}}(t)} \times q_{R_{S L, \bar{k}}(t)}} d t}\right) .
\end{aligned}
$$

Note that $\delta_{B}\left(q_{R_{S U, \bar{k}}(t)}, q_{R_{S L, \bar{k}}(t)}\right)$ is definitely no less than $\delta_{B}\left(q_{R_{S U}(t)}, q_{R_{S L}(t)}\right)$ because the reliability bound by fusing all the experts' MSII is no wider than that by fusing the MSII without the $k$ th expert. Therefore, $I_{B}\left(\mathbf{m}_{k}\right)$ is a non-negative measure. Particularly, $I_{B}\left(\mathbf{m}_{k}\right)=0$ means the $k$ th expert's MSII provides no information for system reliability estimate, i.e., the BBA of the $k$ th expert is vacuous. A greater value of $I_{B}\left(\mathbf{m}_{k}\right)$ indicates the MSII from the $k$ th expert contains more new information with respect to system reliability estimates.

\section{APPLICATIONS}

In this section, two applications of the developed methods are presented. The first is the flow transmission system illustrated in Section II.A to demonstrate the main procedures of the developed conflict quantification approaches. The second is an engineering case study on a cutter feeding control system of CNC lathes.

\section{A. A Numerical Example}

The flow transmission system, as shown in Fig. 1, is chosen for the numerical case study [14]. The reliability function of the
Step 2: Evaluate the system reliability bounds $\left[R_{S L, \bar{k}}(t), R_{S U, \bar{k}}(t)\right]$ by fusing the MSII without the $k$ th experts via Eq. (5), and then, calculating the corresponding PDFs of the upper and lower system lifetime distributions, denoted as $q_{R_{S U, \bar{k}}(t)}$ and $q_{R_{S L, \bar{k}}(t)}: q_{R_{S U, \bar{k}}(t)}=-d R_{S U, \bar{k}}(t) / d t$ and $q_{R_{S L, \bar{k}}(t)}=$ $-d R_{S L \bar{k}}(t) / d t$.

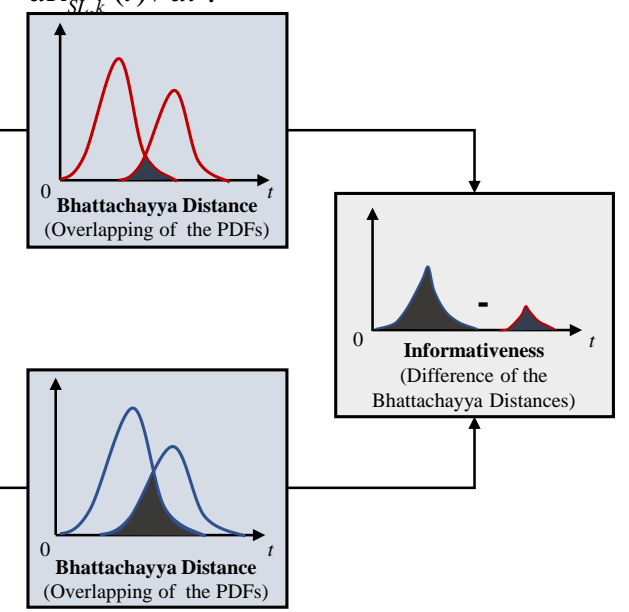

flow transmission system is defined as Eq. (3). To assess the system reliability over time, the MSII is elicited from four experts. Expert 1 provided the interval-valued state probabilities of all the pipes at time $t=100$ hours. Expert 2 provided the interval-valued state probabilities of the system at time $t=150$ hours. Expert 3 gave the interval-valued sojourning

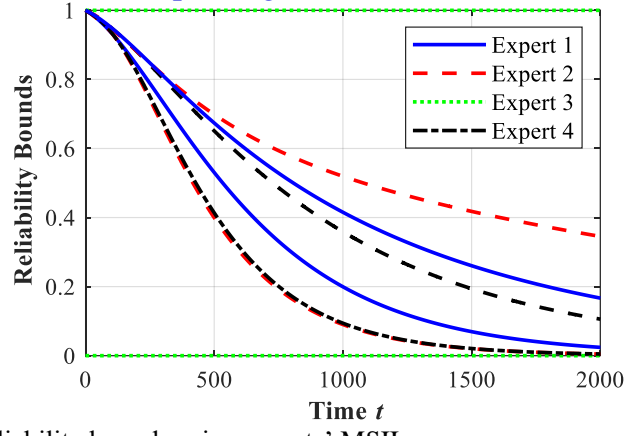

Fig. 6. Reliability bounds using experts' MSII.

TABLE V

THE MSII ELICITED FROM FOUR EXPERTS (TIME UNIT: HOURS)

\begin{tabular}{cc}
\hline \hline Expert ID & Experts'MSII \\
\hline & $0.795 \leq p_{1,2}(100) \leq 0.805,0.090 \leq p_{1,1}(100) \leq 0.110$ \\
1 & $0.096 \leq p_{1,0}(100) \leq 0.104,0.690 \leq p_{2,2}(100) \leq 0.710$ \\
& $0.190 \leq p_{2,1}(100) \leq 0.210,0.090 \leq p_{2,0}(100) \leq 0.110$ \\
& $0.958 \leq p_{3,1}(100) \leq 0.965,0.035 \leq p_{3,0}(100) \leq 0.042$ \\
\hline \multirow{2}{*}{2} & $0.75 \leq p_{S, 6}(150)+p_{S, 5}(150)+p_{S, 4}(150)$ \\
& $+p_{S, 3}(150)+p_{S, 2}(150) \leq 0.85$ \\
\hline \multirow{2}{*}{3} & $160 \leq T_{2,2} \leq 270$ \\
\hline \multirow{2}{*}{4} & $500 \leq M T T F_{1} \leq 600,400 \leq M T T F_{2} \leq 500$, \\
& $2700 \leq M T T F_{3} \leq 2800$ \\
\hline \hline
\end{tabular}

time of Pipe \#2 being in State 2. Expert 4 presented the intervalvalued MTTFs of all the three pipes. The specific experts' MSII are tabulated in Table V. Equation (4) is, then, used to assess the reliability bounds by using different experts' MSII. The 
results, as shown in Fig. 6, indicate that the reliability bounds assessed by the four experts' MSII are not consistent.

The proposed two-dimensional conflict measure is used to quantify and visualize the conflict between the four experts' MSII. The pre-defined threshold of conflict tolerance $\varepsilon$ is set to be 0.3 . Firstly, we quantify the conflict at two specific time instants, $t=100$ hours and $t=500$ hours. Equation (23) is used to calculate the BBAs of the four experts induced from their corresponding reliability bounds at these two time instants, i.e., $\mathbf{m}_{i}(100)$ and $\mathbf{m}_{i}(500)$. The results are given in Table VI. Table VI also presents the combined BBAs via DRC without the $i$ th expert's BBA, i.e., $\mathbf{m}_{\oplus \bar{i}}(100)$ and $\mathbf{m}_{\oplus \bar{i}}(500)$. Then, Eqs.

TABLE VI

THE INDUCED BBAS FROM DIFFERENT EXPERTS' RELIABILITY BOUNDS AT TIME $t=100$ AND $t=500$ HOURS

\begin{tabular}{|c|c|c|}
\hline \multirow{2}{*}{$\begin{array}{c}\text { Expert } \\
\text { ID }\end{array}$} & \multicolumn{2}{|c|}{ The induced BBAs from reliability bounds } \\
\hline & $t=100$ hours & $t=500$ hours \\
\hline \multirow{2}{*}{1} & $\mathbf{m}_{1}(100)=(0,0.9353,0.0500,0.0147)$ & $\mathbf{m}_{1}(500)=(0,0.5306,0.3261,0.1433)$ \\
\hline & $\mathbf{m}_{\oplus \overline{\mathrm{I}}}(100)=(0,0.9955,0.0043,0.0002)$ & $\mathbf{m}_{\oplus \overline{\mathrm{I}}}(500)=(0,0.5216,0.3820,0.0964)$ \\
\hline \multirow{2}{*}{2} & $\mathbf{m}_{2}(100)=(0,0.9364,0.0519,0.0117)$ & $\mathbf{m}_{2}(500)=(0,0.3996,0.3014,0.2990)$ \\
\hline & $\mathbf{m}_{\oplus \overline{2}}(100)=(0,0.9955,0.0043,0.0002)$ & $\mathbf{m}_{\oplus \overline{2}}(500)=(0,0.5952,0.3548,0.0500)$ \\
\hline \multirow{2}{*}{3} & $\mathbf{m}_{3}(100)=(0,0,0,1)$ & $\mathbf{m}_{3}(500)=(0,0,0,1)$ \\
\hline & $\mathbf{m}_{\oplus \overline{3}}(100)=(0,0.9997,0.0003,0)$ & $\mathbf{m}_{\oplus \overline{3}}(500)=(0,0.6420,0.3360,0.0220)$ \\
\hline \multirow[b]{2}{*}{4} & $\mathbf{m}_{4}(100)=(0,0.9364,0.0505,0.0131)$ & $\mathbf{m}_{4}(500)=(0,0.4137,0.3490,0.2373)$ \\
\hline & $\mathbf{m}_{\oplus \overline{4}}(100)=(0,0.9955,0.0044,0.0001)$ & $\mathbf{m}_{\oplus \overline{4}}(500)=(0,0.6029,0.3367,0.0604)$ \\
\hline
\end{tabular}

(18) and (20) are used to calculate the conflict factor and Jousselme distance at these two time instants, respectively. The proposed two-dimensional conflict measure is presented in Figs. 7(a) and 7(b). As shown in Fig. 7(a), at time $t=100$ hours, the induced BBAs of Experts 1, 2, and 4 are almost the same, therefore, the Jousselme distances between these three BBAs are close to zero while the conflict factors are still non-negative as the three experts' BBAs have non-negative masses at the propositions $\{W\}$ and $\{F\}$. Moreover, the conflict factor of the BBA of Expert 3 with respect to all the other experts' BBAs are zero because the BBA of Expert 3 is vacuous as shown in Table VI. This result is also accordance with the fourth property of the average conflict factor, that is, the vacuous BBA should not conflict with any BBAs. As shown in Fig. 7(b), the twodimensional conflict measure between any two experts among Experts 1,2, and 4 all lie in region $B$. This is because the BBAs of Experts 1, 2 and 4 at time $t=500$ hours are almost evenly distributed on the singletons, as shown in Table V. Additionally, Eqs. (25) and (26) are used to calculate the total average conflict factors and the total average Jousselme distances at these two time instants, respectively. The results, as presented in Figs. 7(c) and 7(d), show that the total average conflict factor of the BBA of Expert 3 with respect to $\mathbf{m}_{\oplus \overline{3}}(100)$ and $\mathbf{m}_{\oplus \overline{3}}(500)$ are both zero because the BBA of
Expert 3 is vacuous. Moreover, as shown in Fig. 7(c), the total two-dimensional conflict measures of Experts 1, 2, and 4 at time $t=100$ hours are almost the same because $\mathbf{m}_{\oplus \overline{1}}(100)$, $\mathbf{m}_{\oplus \overline{2}}(100)$, and $\mathbf{m}_{\oplus \overline{4}}(100)$ are the same as shown in Table V. It indicates that both the combined BBAs via DRC and each individual BBA of Experts 1, 2, and 4 provide the same information: the system is working at time $t=100$ hours. As shown in Fig. 7(d), the total two-dimensional conflict measures of Experts 1, 2, and 4 indicate that the masses of these three experts are nearly distributed evenly on the singletons. These results substantiate the argument that the proposed twodimensional conflict measure can effectively quantify and visualize the conflict among the BBAs induced by experts' MSII.

To demonstrate the superiority of the two-dimensional conflict measure in quantify the conflict between BBAs with respect to other conflict measures in the DST, the conflict of the four experts' BBAs at both $t=100$ and $t=500$ hours are calculated by the conflict factor, the Jousselme distance, the cosine measure, and the supremum norm of the difference of betting commitment via Eqs. (18), (20), (16), and (17), respectively. The comparative results of the conflict between any pairs of BBAs and the total conflict of each expert's BBAs at both $t=100$ and $t=500$ hours are presented in Table VII. As shown in Table VII, all these five conflict measures indicate the conflicts among the BBAs of Experts 1, 2, and 4 at $t=100$ hours are small as the induced BBAs of Experts 1,2, and 4 are almost the same. However, as for the BBA of Expert 3, the values of cosine measure, Jousselme distance, and supremum norm of the difference of betting commitment from both the mutual conflict and total conflict perspectives are greater than the threshold of conflict tolerance 0.3. It is, therefore, concluded that all the three distance-based conflict measures are incapable of handling the conflict quantification of a vacuous BBA with arbitrary BBA. As shown from Table VII, the mutual conflict of Expert 3 with any other experts by the proposed twodimensional measure is $\langle 0,0.6623\rangle$, which is located in region $C$. It means that one of the two experts provided very limited information to the reliability assessment. This result is accordance with the fact as the BBA of Expert 3 is vacuous. Thereby, the two-dimensional measure is superior to the conflict factor and the distance-based conflict measures.

Furthermore, the proposed conflict measure is used to quantify the conflict between experts' MSII in the entire time horizon, i.e., $T_{\mathrm{int}}=\left[T_{L}, T_{U}\right]=[0,2000]$. We use the average twodimensional conflict measure to quantify the total conflict of these four experts' MSII. By Eq. (23), we can obtain the BBAs of all the experts during the time interval $T_{\text {int }}$. Then, Eqs. (18) and (20) are utilized to calculate the instantaneous conflict factor and Jousselme distance, and the results are given in Fig. 8. As shown in Figs. 8(a) and 8(c), the conflict factors involving Experts 1, 2, and 4 all start from zero, reach their maximum values, and finally back to zero. This is because all these

TABLE VII

COMPARATIVE STUDIES OF THE CONFLICT MEASURES

\begin{tabular}{|c|c|c|c|c|c|c|c|c|c|c|}
\hline \multirow[b]{2}{*}{ Experts } & \multicolumn{5}{|c|}{$t=100$ hours } & \multicolumn{5}{|c|}{$t=500$ hours } \\
\hline & $k_{i \oplus i}$ & $J D\left(\mathbf{m}_{i}, \mathbf{m}_{i}\right)$ & $1-\cos \left(\mathbf{m}_{i}, \mathbf{m}_{j}\right)$ & $\operatorname{diffBet} P_{\mathbf{m}_{j}}^{\mathbf{m}_{i}}$ & $\operatorname{TDCM}\left(\mathbf{m}_{i}, \mathbf{m}_{j}\right)$ & $k_{i \oplus j}$ & $J D\left(\mathbf{m}_{i}, \mathbf{m}_{j}\right)$ & $1-\cos \left(\mathbf{m}_{i}\right.$ & $\underset{j}{\operatorname{djffBet}} \mathrm{P}_{\mathbf{m}_{j}}^{\mathbf{m}_{i}}$ & $\operatorname{TDCM}\left(\mathbf{m}_{i}, \mathbf{m}_{j}\right)$ \\
\hline $1 \& 2$ & 0.0953 & 0.0016 & 0 & 0.0005 & $<0.0953,0.0016>$ & 0.2903 & 0.0942 & 0.0522 & 0.0531 & $<0.2903,0.0942>$ \\
\hline $1 \& 3$ & 0 & 0.6623 & 0.9843 & 0.4427 & $<0,0.6623>$ & 0 & 0.4404 & 0.7758 & 0.1022 & $<0,0.4404>$ \\
\hline $1 \& 4$ & 0.0940 & 0.0008 & 0 & 0.0003 & $<0.0940,0.0008>$ & 0.3201 & 0.0842 & 0.0274 & 0.0699 & $<0.3201,0.0842>$ \\
\hline
\end{tabular}




\begin{tabular}{|c|c|c|c|c|c|c|c|c|c|c|}
\hline $2 \& 3$ & 0 & 0.6631 & 0.9876 & 0.4422 & $<0,0.6631>$ & 0 & 0.3539 & 0.4872 & 0.0491 & $<0,0.3539>$ \\
\hline $2 \& 4$ & 0.0959 & 0.0010 & 0 & 0.0007 & $<0.0959,0.0010>$ & 0.2642 & 0.0350 & 0.0090 & 0.0168 & $<0.2642,0.0350>$ \\
\hline $3 \& 4$ & 0 & 0.6631 & 0.9856 & 0.4430 & $<0,0.6631>$ & 0 & 0.3827 & 0.5984 & 0.0323 & $<0,0.3827>$ \\
\hline $1 \& \overline{1}$ & 0.0538 & 0.0534 & 0.0013 & 0.0529 & $<0.0538,0.0534>$ & 0.3728 & 0.0400 & 0.3727 & 0.0324 & $<0.3728,0.0400>$ \\
\hline $2 \& \overline{2}$ & 0.0558 & 0.0536 & 0.0014 & 0.0533 & $<0.0558,0.0536>$ & 0.3212 & 0.1434 & 0.3212 & 0.0711 & $<0.3212,0.1434>$ \\
\hline $3 \& \overline{3}$ & 0 & 0.7069 & 1 & 0.4997 & $<0,0.7069>$ & 0 & 0.5124 & 0 & 0.1529 & $<0,0.5124>$ \\
\hline $4 \& \overline{4}$ & 0.0543 & 0.0530 & 0.0013 & 0.0526 & $<0.0543,0.0530>$ & 0.3497 & 0.1341 & 0.3497 & 0.1007 & $<0.3497,0.1341>$ \\
\hline
\end{tabular}
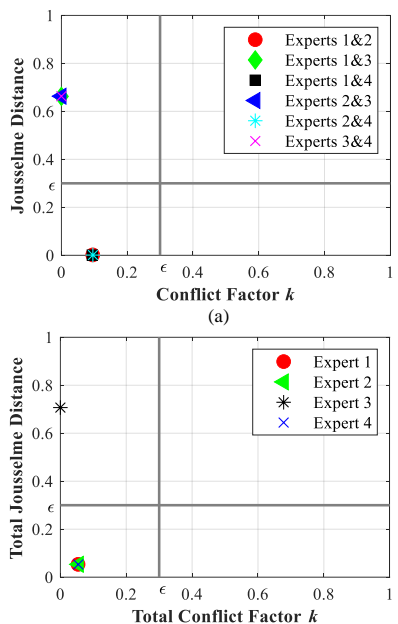

(c)

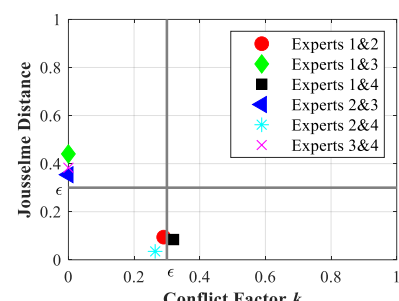

(b)

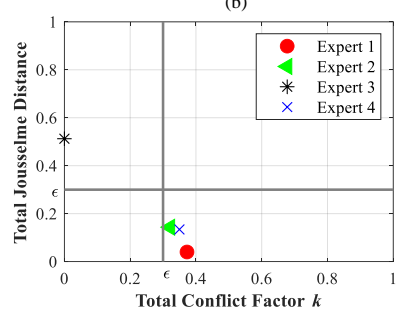

(d)

Fig. 7. The two-dimensional conflict measures of experts' MSII. (a) Mutual conflict quantification at time $t=100$ hours; (b) Mutual conflict quantification at time $t=150$ hours; (c) Total conflict quantification at time $t=100$ hours; (d) Total conflict quantification at time $t=150$ hours.

experts believed that the mass of the system failure at $t=0$ is zero and the mass of the system working at time $t=2000$ hours is zero. The conflict factors involving Expert 3 remain zero as the BBA of Expert 3 is vacuous over the time horizon $T_{\text {int }}=[0,2000]$. Moreover, as shown in Figs. 8(b) and 8(d), the Jousselme distance of the BBA of Expert 3 with respect to that of the other experts all start from $\sqrt{1 / 2}$. It can be directly calculated by the Jousselme distance of a vacuous BBA and a categorical BBA, that is:

$$
\sqrt{\quad \sqrt{\frac{1}{2}(0,-1,0,1)\left[\begin{array}{cccc}
1 & 0 & 0 & 0 \\
0 & 1 & 0 & 0.5 \\
0 & 0 & 1 & 0.5 \\
0 & 0.5 & 0.5 & 1
\end{array}\right](0,-1,0,1)^{T}}=\sqrt{1 / 2}}
$$

Moreover, the Jousselme distances of the BBA of Expert 3 with respect to that of the other experts go to $\sqrt{1 / 2}$ when the system becomes very aged. Based on the instantaneous conflict factors and Jousselme distances, the average conflict factors and average Jousselme distances can be calculated by Eqs. (19) and (22), respectively, while the total average conflict factors and average Jousselme distances can be calculated by Eqs. (25) and (26), respectively. The resulting two-dimensional conflict measure $\operatorname{TDCM}\left(\mathbf{m}_{i}, \mathbf{m}_{j}\right)$ and total two-dimensional conflict measure $\operatorname{TTDCM}\left(\mathbf{m}_{i}, \mathbf{m}_{\oplus \bar{i}}\right)$ are illustrated in Fig. 9. The results of the two-dimensional conflict measure $\operatorname{TDCM}\left(\mathbf{m}_{i}, \mathbf{m}_{j}\right)$ between any two experts in Experts 1, 2, and 4 show that the MSII from these three experts are not in conflict. Because there is a large region of intersection among their reliability bounds, as shown in Fig. 6. On the other hand, the total two-dimensional

conflict measure results of Experts 1, 2, and 4, i.e., $\operatorname{TTDCM}\left(\mathbf{m}_{1}, \mathbf{m}_{\oplus \overline{1}}\right) \quad, \quad \operatorname{TTDCM}\left(\mathbf{m}_{2}, \mathbf{m}_{\oplus \overline{2}}\right) \quad, \quad$ and $\operatorname{TTDCM}\left(\mathbf{m}_{4}, \mathbf{m}_{\oplus \overline{4}}\right)$ also substantiate that these three experts' MSII are not in conflict. The total two-dimensional conflict measure of Expert 3, i.e., $\operatorname{TTDCM}\left(\mathbf{m}_{3}, \mathbf{m}_{\oplus \overline{3}}\right)$, is located in region $C$. It indicates that even though the BBA of Expert 3 is not in conflict with that of the other experts, it provides nothing to the system reliability estimate.
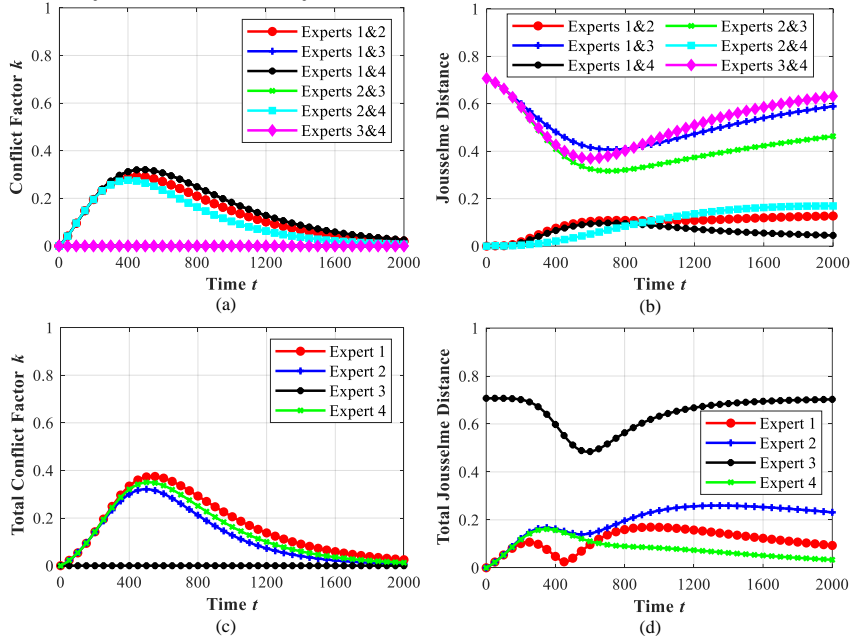

Fig. 8. The instantaneous conflict factor $k_{i \oplus j}(t)$, instantaneous Jousselme distance $J D\left(\mathbf{m}_{i}(t), \mathbf{m}_{j}(t)\right)$, instantaneous total conflict factor $k_{\mathbf{m}_{i} \oplus \mathbf{m}_{\oplus \bar{i}}}(t)$, and instantaneous total Jousselme distance $J D\left(\mathbf{m}_{i}(t), \mathbf{m}_{\oplus \bar{i}}(t)\right)$.
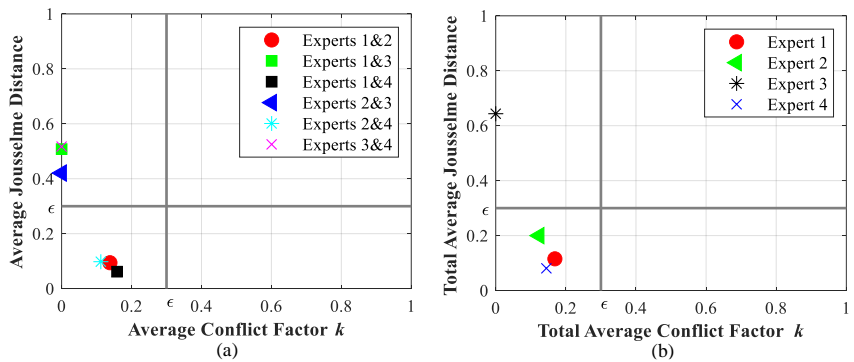

Fig. 9. The two-dimensional conflict measure over time interval [0, 2000] . (a) Mutual conflict quantification. (b) Total conflict quantification.

As concluded from the above analysis, the conflict measures only visualize the conflict among the experts' MSII but are not able to quantify the informativeness of experts' MSII to system reliability estimate. Therefore, the Bhattacharyya distancebased informativeness measure is also calculated. By converting all experts' MSII into constraints of the transition intensities of the three components, the narrowest reliability bound can be determined by Eq. (5), while the corresponding Bhattacharyya distance $\delta_{B}\left(q_{R_{S U}(t)}, q_{R_{S L}(t)}\right)$ can be evaluated by Eq. (28). The result is shown in Table VIII. In the same fashion, $\delta_{B}\left(q_{R_{S U, \bar{K}}(t)}, q_{R_{S L, \bar{k}}(t)}\right)$ can be also evaluated via Eq. (29) for the reliability bounds without the $k$ th $(k=1,2,3,4)$ expert's MSII. 
The results are given in Table VIII. Therefore, the Bhattacharyya distance-based informativeness of the $k$ th expert's MSII $I_{B}\left(\mathbf{m}_{k}\right)$ can be determined through Eq. (30). The results are tabulated in Table IX. As indicated in Table IX, although the conflict factor of Expert 3 is lower than the others, it is not very useful, as it provides the least information as indicated by the informativeness measure. On the contrary, Expert 1 has the highest total average conflict factor against the others, as shown in Fig. 9(b), but provides the greatest information to the system reliability estimate.

Based on the conflict and informativeness results, the consensus on final reliability estimate of the flow transmission system can be achieved by calibrate the judgements from Expert 3 or get rid of the judgements from Expert 3. This is because the judgements from Experts 1, 2, and 4 contribute to TABLE VIII

THE BHATTACHARYYA DISTANCE OF $\delta_{B}\left(q_{R_{S U}(t)}, q_{R_{S L}(t)}\right)$ AND

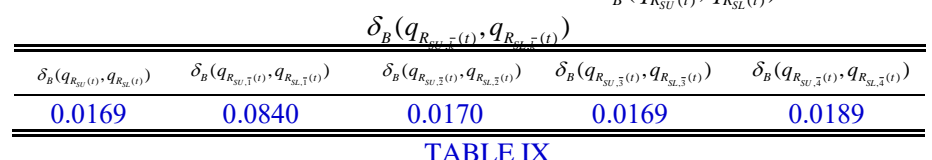

THE BHATTACHARYYA DISTANCE-BASED INFORMATIVENESS OF THE EXPERTS' MSII.

\begin{tabular}{ccccc}
\hline \hline & Expert 1 & Expert 2 & Expert 3 & Expert 4 \\
\hline$I_{B}\left(\mathbf{m}_{k}\right)$ & 0.0671 & 0.0010 & 0 & 0.0020 \\
\hline \hline
\end{tabular}

the final reliability estimate while they are not in conflict with that of the other experts. As for Expert 2, even his/her judgement is not conflicting with the others, it has little effect on the final reliability estimate because the reliability bound from Expert 2 is relatively wide. Therefore, Expert 2 should further narrower down his/her judgement so as to contribute to the reliability estimate. Expert 3 should express more reliability-related information on this system, otherwise, the current judgement from Expert 3 can be omitted.

\section{B. A Cutter Feeding Control System}

We also apply the two-dimensional conflict measure on a realworld large engineering system, i.e., a cutter feeding control system of DL series CNC lathes, as shown in Fig 10. For the cutter feeding control system, a signal generated by 611D-type servo driven module $(M O)$ is transmitted through electric wire $(E W)$ to control the motor $(M T)$, and a speed feedback device $(S F)$ is used to return the speed signal to servo driven module $(M O)$, while the grating scales $(G R)$ report the straightness to $M O$ so as to adjust the feeding speed and direction [11]. The components $E W, G R$, and $M O$ are binary components as they are electronic devices. The components $M T$ and $S F$ are regarded as four-state components, say States $0,1,2$, and 3, as they all contain electric motors which can work at two intermediate states in-between perfectly functioning and completely failed states. The components $M T$ and $S F$ are assembled together and can be viewed as subsystem $S_{1}$. Based on the functional relations of all the components to the cutter feeding control system, the entire system also possesses four states, say States $0,1,2$, and 3 . The states of the system with respect to each combination of components' states are tabulated in Table X. A complete version of Table $X$ consists of $2 \times 2 \times 2 \times 4 \times 4=128$ rows, corresponding to all the possible components' states combinations. Based on Table $\mathrm{X}$, the relation among the components' states and the system states can be completely determined.

The system is deemed as failure if its state being in the unacceptable state 0 , i.e., $\mathbf{D}=\{0\}$. Therefore, the system reliability can be formulated as the probability that the state of the cutter feeding control system is not less than State 1, and it can be mathematically written as:

$$
\begin{aligned}
& R_{S}(t)=1-F_{S}(t)=1-\sum_{i \in \mathbf{D}} p_{S, i}(t) \\
& =p_{E W, 1}(t) \times p_{G R, 1}(t) \times p_{M O, 1}(t) \times\left(\sum_{i>0} \sum_{j>0}\left(p_{M T, i}(t) \times p_{S F, j}(t)\right)\right)
\end{aligned}
$$

Fig. 10 The cutter feeding control system of DL series CNC lathes [10],[11].

TABLE X

THE STATES OF THE $X$ FEEDING CONTROL SYSTEM AND ITS STATE COMBINATIONS OF COMPONENTS.

\begin{tabular}{cccccc}
\hline \hline $\begin{array}{c}\text { State } \\
\text { of } \\
\text { system }\end{array}$ & $\begin{array}{c}\text { State of } \\
\text { component } \\
E W\end{array}$ & $\begin{array}{c}\text { State of } \\
\text { component } \\
G R\end{array}$ & $\begin{array}{c}\text { State of } \\
\text { component } \\
M O\end{array}$ & $\begin{array}{c}\text { State of } \\
\text { component } \\
M T\end{array}$ & $\begin{array}{c}\text { State of } \\
\text { component } \\
S F\end{array}$ \\
\hline 0 & 0 & 0 & 0 & 0 & 0 \\
0 & 0 & 0 & 0 & 0 & 1 \\
0 & 0 & 0 & 0 & 0 & 2 \\
0 & 0 & 0 & 0 & 0 & 3 \\
0 & 0 & 0 & 0 & 1 & 0 \\
$\vdots$ & $\vdots$ & $\vdots$ & $\vdots$ & $\vdots$ & $\vdots$ \\
2 & 1 & 1 & 1 & 2 & 3 \\
0 & 1 & 1 & 1 & 3 & 0 \\
1 & 1 & 1 & 1 & 3 & 1 \\
2 & 1 & 1 & 1 & 3 & 2 \\
3 & 1 & 1 & 1 & 3 & 3 \\
\hline \hline
\end{tabular}
TABLE XI

THE THREE EXPERTS' MSII OF THE CUTTER FEEDING CONTROL SYSTEM (TIME UNIT: MONTHS).

\begin{tabular}{cc}
\hline \hline $\begin{array}{c}\text { Expert } \\
\text { ID }\end{array}$ & Experts' MSII \\
& $0.60 \leq p_{S F, 3}(10) \leq 0.64,0.12 \leq p_{S F, 2}(10) \leq 0.14$, \\
& $0.11 \leq p_{S F, 1}(10) \leq 0.15,0.13 \leq p_{S F, 0}(10) \leq 0.17$, \\
& $0.80 \leq p_{M T, 3}(10) \leq 0.82,0.06 \leq p_{M T, 2}(10) \leq 0.10$, \\
1 & $0.04 \leq p_{M T, 1}(10) \leq 0.08,0.06 \leq p_{M T, 0}(10) \leq 0.10$, \\
& $0.90 \leq p_{M O, 1}(10) \leq 0.93,0.07 \leq p_{M O, 0}(10) \leq 0.10$, \\
& $0.86 \leq p_{G R, 1}(10) \leq 0.88,0.12 \leq p_{G R, 0}(10) \leq 0.14$, \\
& $0.95 \leq p_{E W, 1}(10) \leq 0.96,0.04 \leq p_{E W, 0}(10) \leq 0.05$ \\
2 & $0.70 \leq R_{S}(15) \leq 0.80$ \\
3 & $15 \leq M T T F_{S_{1}} \leq 30,150 \leq M T T F_{M O} \leq 200$, \\
& $150 \leq M T T F_{G R} \leq 200,180 \leq M T T F_{E W} \leq 200$ \\
\hline \hline
\end{tabular}

Three experts are invited to express their judgments on the reliability-related information of the system. Their uncertainties are expressed as interval values. The collected MSII are 
tabulated in Table XI. Expert 1 presented the interval-valued state probabilities of all components at time $t=10$ months. The interval-valued system reliability at time $t=15$ months is provided by Expert 2, while Expert 3 expressed the intervalvalued MTTF of the subsystem $S_{1}$ and the components $M O$, $G R$, and $E W$. Thereby, the reliability bound induced from each expert's MSII can be evaluated via Eq. (4), and the results are shown in Fig. 11. As shown in Fig. 11, the reliability bounds induced from Expert 1 are more precise than that of Expert 3, whereas the reliability bounds induced from Expert 2 are totally inconsistent with these of the other two experts.

The experts are interested in the conflict of their MSII on the entire time horizon, i.e., $T_{\mathrm{int}}=\left[T_{L}, T_{U}\right]=[0,35]$ months. The instantaneous conflict factor and Jousselme distance between

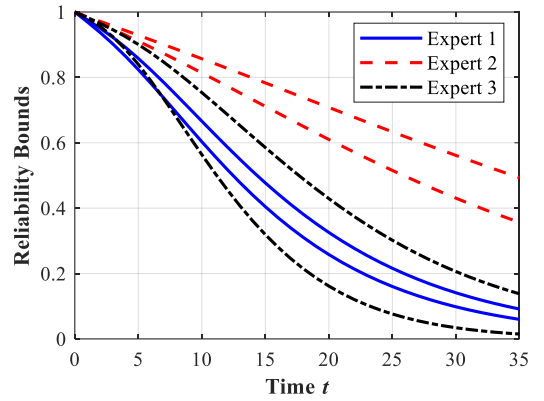

Fig. 11. Reliability bounds of the cutter feeding control system using the three experts' MSII.

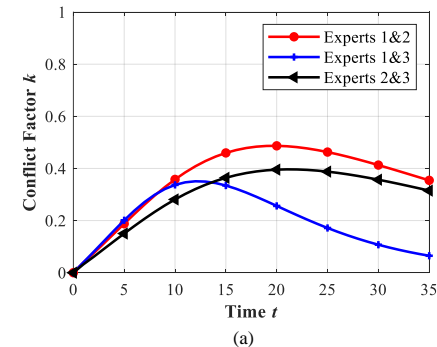

(a)

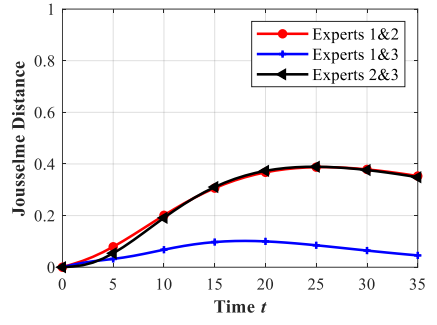

(b)
Fig. 12. The instantaneous conflict factor $k_{i \oplus j}(t)$ and Jousselme distance $J D\left(\mathbf{m}_{i}(t), \mathbf{m}_{j}(t)\right)$

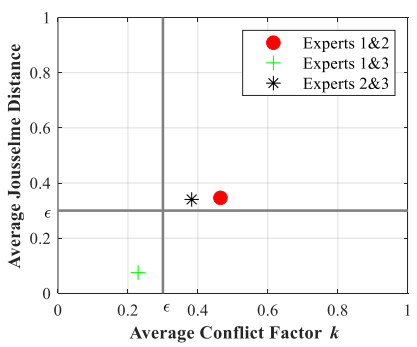

(a)

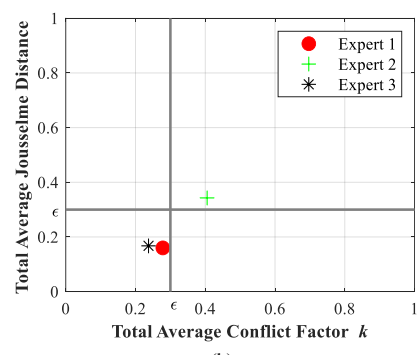

(b)
Fig. 13. The average two-dimensional conflict measure. (a) Mutual conflict. (b) Total conflict.

any two experts' BBAs are calculated by Eqs. (18) and (20), respectively. The results, as shown in Fig. 12, indicate that the minimum conflict among experts' BBAs exists when the system is brand new. Moreover, the conflict factor between Experts 1 and 2 is always larger than that of Experts 2 and 3 because the reliability bounds of Expert 1 are more precise than these of Expert 3. Such a result also accords with the fourth property of the average conflict factor, i.e., imprecision monotonicity. Furthermore, the average conflict factors and average Jousselme distances can be calculated by Eqs. (19) and (22), respectively, while the total average conflict factors and average Jousselme distances can be calculated by Eq. (25) and (26), respectively. The resulting two-dimensional conflict measure $\operatorname{TDCM}\left(\mathbf{m}_{i}, \mathbf{m}_{j}\right)$ and total two-dimensional conflict measure $\operatorname{TTDCM}\left(\mathbf{m}_{i}, \mathbf{m}_{\oplus \bar{i}}\right)$ are illustrated in Fig. 13. As shown in Fig. 13(a), the two-dimensional conflict measure involving Expert 2, i.e., $\operatorname{TDCM}\left(\mathbf{m}_{1}, \mathbf{m}_{2}\right)$ and $\operatorname{TDCM}\left(\mathbf{m}_{2}, \mathbf{m}_{3}\right)$ indicate that the judgement of Expert 2 is conflicting with the other two experts, while the judgement of Expert 1 is not conflicting with Expert 3. Moreover, from the perspective of total conflict, it also substantiates that only the judgement of Expert 2 is conflicting with the other two experts. Therefore, the proposed two-dimensional conflict measure can effectively quantify and visualize the conflict of the three experts' MSII. Based on both the mutual conflict and total conflict results, further calibration of the experts' MSII can be done by adjusting the judgement of Expert 2. Specifically, as Expert 2 takes an optimistic attitude on the system reliability at time $t=15$ months, the calibration of the judgement of Expert 2 should shift toward a more conservative reliability estimate which is oftentimes preferable in engineering practices.

From the above analysis of two illustrative examples, the distinguishing characteristics of our proposed method are listed as follows:

(1) The novel framework of the conflict quantification of experts' MSII allows experts to provide the imprecise reliability-related judgements from multiple physical hierarchies of an MSS at different times instants.

(2) The proposed two-dimensional conflict measure is an effective tool to manipulate the conflict quantification of any pairs of BBAs under the DST framework.

(3) Via the Bhattacharyya distance-based informativeness method, the individual contribution of an expert's judgement to the system reliability assessment is evaluated, which is critical for the further calibration of experts' judgements and the refinement of the experts.

\section{CONCLUSIONS AND FUTURE WORKS}

In this article, a two-dimensional conflict measure was proposed to quantify the conflict between experts' MSII for system reliability assessment. The two dimensions of the conflict measure were characterized by the average conflict factor and the average Jousselme distance, respectively. To apply the conflict measure, first, the reliability estimate induced from each expert's MSII was used to construct the corresponding basic belief assignment (BBA) under the DST. Then, the average conflict factor and average Jousselme distance in DST were calculated to characterize the two dimensions of conflict, respectively. The conflict quantification by the two-dimensional conflict measure involved mutual conflict quantification and total conflict quantification. Moreover, a Bhattacharyya distance-based informativeness method was developed to further quantify the informativeness of a specific expert's MSII to the system reliability estimate. As concluded from the two illustrative examples, the proposed two-dimensional conflict measure is superior to the separate one, i.e., the conflict factor or the Jousselme distance, in quantifying the conflict among experts' MSII. 
It bears noting that the proposed method is still a preliminary work for system reliability assessment by utilizing experts' MSII. Further research is needed on how to achieve the consensus on reliability estimate by experts' MSII based on the developed conflict measures. This could be done by formulating an optimization model in which the objective is to minimize the two-dimensional conflict measure while the decision variables are the range of the experts' MSII. In that way, the final reliability estimate with the minimum conflict of experts' MSII can be achieved and viewed as a rational consensus on system reliability estimate. Moreover, the threshold of conflict tolerance is fixed in this work, it is interesting to find the relation of this threshold with the conflict factor, the Jousselme distance, and also the number of experts under the close-world assumption. Therefore, the threshold of conflict tolerance can be determined by the BBAs induced from experts' MSII rather in a subjective manner. Lastly, both the conflict measures and the Bhattacharyya distance-based informativeness method can be further used as criteria for the calibration of experts' judgements and the refinement of experts.

\section{APPENDIX A}

The proofs of the four properties of the proposed average conflict factor are provided as follows.

Proof of Property 1: As the conflict factor $k_{i \oplus j}(t)$ at any time $t$ is bounded, i.e, $0 \leq k_{i \oplus j}(t) \leq 1$, the average conflict factor $k_{i \oplus j}=m_{i \oplus j}^{\varnothing}=\frac{1}{T-T_{T}} \int k_{i \oplus j}(t) d t$ is also bounded, i.e.,

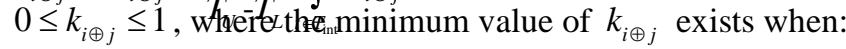

$$
k_{i \oplus j}=0
$$$$
\Leftrightarrow \frac{1}{T_{U}-T_{L}} \int_{t \in T_{\text {int }}} \sum_{A \cap B=\varnothing, A, B \subseteq \Omega} m_{i}^{A}(t) m_{j}^{B}(t) d t=0
$$$$
\Leftrightarrow \forall t \in T_{\text {int }}, m_{i}^{\{W\}}(t)=m_{j}^{\{W\}}(t)=0 \text { or } \quad m_{i}^{\{F\}}(t)=m_{j}^{\{F\}}(t)=0
$$

When $m_{i}^{\{W\}}(t)=m_{j}^{\{W\}}(t)=0$, we have $\mathbf{m}_{i}(t)=\left(0,0, s_{1}, 1-s_{1}\right)$ and $\mathbf{m}_{j}(t)=\left(0,0, s_{2}, 1-s_{2}\right)$ with $s_{1}$ and $s_{2}$ are two random values range from 0 to 1 . When $m_{i}^{\{F\}}(t)=m_{j}^{\{F\}}(t)=0$, we have $\mathbf{m}_{i}(t)=\left(0, s_{1}, 0,1-s_{1}\right)$ and $\mathbf{m}_{j}(t)=\left(0, s_{2}, 0,1-s_{2}\right)$. It can be seen that in the above two scenarios, both $\mathbf{m}_{i}(t)$ and $\mathbf{m}_{j}(t)$ are simple BBAs with the same focal elements. The maximum value of $k_{i \oplus j}$ exists when:

$$
\begin{aligned}
k_{i \oplus j}=1 \Leftrightarrow & \frac{1}{T_{U}-T_{L}} \int_{t \in T_{\mathrm{int}}} \sum_{A \cap B=\varnothing, A, B \subseteq \Omega} m_{i}^{A}(t) m_{j}^{B}(t) d t=1 \\
\Leftrightarrow & \forall t \in T_{\mathrm{int}}, A \neq B \neq\{W, F\}, \\
& \text { and } m_{i}^{A}(t)=m_{j}^{B}(t)=1
\end{aligned}
$$

where $A \neq B \neq\{W, F\}$ means $A=\{W\}, B=\{F\}$ or $A=\{F\}$, $B=\{W\}$. The proof is completed.

Proof of Property 2: $k_{i \oplus j}=k_{j \oplus i} \Leftrightarrow k_{i \oplus j}-k_{j \oplus i}=0$ where:

$$
\begin{aligned}
k_{i \oplus j}-k_{j \oplus i}= & \frac{1}{T_{U}-T_{L}} \int_{t \in T_{\mathrm{int}}} \sum_{A \cap B=\varnothing, A, B \subseteq \Omega} m_{i}^{A}(t) m_{j}^{B}(t) d t \\
& -\frac{1}{T_{U}-T_{L}} \int_{t \in T_{\mathrm{Tit}}} A \cap B=\varnothing, A, B \subseteq \Omega \\
= & \frac{1}{T_{U}-T_{L}} \int_{t \in T_{\mathrm{int}}} \sum_{A \cap B=\varnothing}\left(m_{i}^{A}(t) m_{i}^{B}(t) d t\right. \\
= & 0
\end{aligned}
$$

which completes the proof.

Proof of Property 3: Suppose that the $k$ th expert provides a piece of more precise information, then a narrower reliability bound, represented by $\left[R_{S L, k^{\prime}}(t), R_{S U, k^{\prime}}(t)\right]$, can be achieved via Eq. (4), where $R_{S L, k^{\prime}}(t)>R_{S L, k}(t)$ and $R_{S U k^{\prime}}(t)<R_{S U, k}(t)$. The corresponding BBA is $\mathbf{m}_{k^{\prime}}(t)=\left(m_{k^{\prime}}^{\varnothing}(t), m_{k^{\prime}}^{\{W\}}(t), m_{k^{\prime}}^{\{F\}}(t)\right.$, $\left.m_{k^{\prime}}^{\{W, F\}}(t)\right)=\left(0, R_{S L, k^{\prime}}(t), 1-R_{S U, k^{\prime}}(t), R_{S U, k^{\prime}}(t)-R_{S L, k^{\prime}}(t)\right) \quad$ with $m_{k^{\prime}}^{\{W\}}(t)>m_{k}^{\{W\}}(t)$ and $m_{k^{\prime}}^{\{F\}}(t)>m_{k}^{\{F\}}(t)$. Let the average conflict factor of $\mathbf{m}_{k}^{\prime}(t)$ with respect to any other BBA $\mathbf{m}_{i}(t)$ be $k_{i \oplus k^{\prime}}$, then one has $k_{i \oplus k}-k_{i \oplus k^{\prime}}<0$, which is detailed in Eq. (A.4) at the bottom of this page. Therefore, we have $k_{i \oplus k^{\prime}}>k_{i \oplus k}$ , which completes the proof.

Proof of Property 4: Let $\mathbf{m}_{i}(t)=\left(m_{i}^{\varnothing}(t), m_{i}^{\{W\}}(t), m_{i}^{\{F\}}(t)\right.$, $\left.m_{i}^{\{W, F\}}(t)\right)=(0,0,0,1)$ be a vacuous BBA, the average conflict factor of $\mathbf{m}_{i}(t)$ with respect to any other expert's BBA is:

$$
\begin{aligned}
k_{i \oplus j} & =\frac{1}{T_{U}-T_{L}} \int_{t \in T_{\text {Tit }}} \sum_{A \cap B=\varnothing, A, B \subseteq \Omega} m_{i}^{A}(t) m_{j}^{B}(t) d t \\
& =\frac{1}{T_{U}-T_{L}} \int_{t \in T_{\text {int }}}\left(m_{i}^{\{W\}}(t) m_{j}^{\{F\}}(t)+m_{i}^{\{F\}}(t) m_{j}^{\{W\}}(t)\right) d t \\
& =0
\end{aligned}
$$

which completes the proof.

\section{APPENDIX B}

The proofs of the four properties of the proposed average Jousselme distance are provided as follows.

Proof of Property 5: As demonstrated in [32], the Jaccard matrix Jac in the Jousselme distance is definiteness. Thereby, the quadratic form $\left(\mathbf{m}_{i}(t)-\mathbf{m}_{j}(t)\right) \mathbf{J a c}\left(\mathbf{m}_{i}(t)-\mathbf{m}_{j}(t)\right)^{T}$ is nonnegative. One has $J D\left(\mathbf{m}_{i}(t), \mathbf{m}_{j}(t)\right) \geq 0$. As for the maximum value of $J D\left(\mathbf{m}_{i}, \mathbf{m}_{j}\right)$, Jousselme et al. [25] proved that $J D\left(\mathbf{m}_{i}(t), \mathbf{m}_{j}(t)\right) \leq 1$, therefore, the proposed average Jousselme distance is also bounded, i.e., $0 \leq J D\left(\mathbf{m}_{i}, \mathbf{m}_{j}\right) \leq 1$.

Proof of Property 6: As the Jaccard matrix $\mathbf{J a c}$ in the Jousselme distance is definiteness, $J D\left(\mathbf{m}_{i}(t), \mathbf{m}_{j}(t)\right)=0$ holds if and only if $\mathbf{m}_{i}(t)-\mathbf{m}_{j}(t)=(0,0,0,0)$, i.e., $\mathbf{m}_{j}(t)=\mathbf{m}_{j}(t)$. $J D\left(\mathbf{m}_{i}(t), \mathbf{m}_{j}(t)\right)=1$ if and only $\left\|\mathbf{m}_{i}(t)-\mathbf{m}_{j}(t)\right\|^{2}$ reaches its maximum, i.e., $\quad \mathbf{m}_{i}(t)=(0,1,0,0) \quad \mathbf{m}_{j}(t)=(0,0,1,0)$, or $\mathbf{m}_{i}(t)=(0,0,1,0), \mathbf{m}_{j}(t)=(0,1,0,0)$, which complete the proof. 


$$
\begin{aligned}
k_{i \oplus k}-k_{i \oplus k^{\prime}} & =\frac{1}{T_{U}-T_{L}} \int_{t \in T_{\mathrm{ith}}} \sum_{A \cap B=\varnothing, A, B \subseteq \Omega} m_{i}^{A}(t) m_{k}^{B}(t) d t-\frac{1}{T_{U}-T_{L}} \int_{t \in T_{\mathrm{int}}} \sum_{A \cap B=\varnothing, A, B \subseteq \Omega} m_{i}^{A}(t) m_{k^{\prime}}^{B}(t) d t \\
& =\frac{1}{T_{U}-T_{L}} \int_{t \in T_{\mathrm{int}}} \sum_{A \cap B=\varnothing, A, B \subseteq \Omega}\left(m_{i}^{A}(t) m_{k}^{B}(t)-m_{i}^{A}(t) m_{k^{\prime}}^{B}(t)\right) d t \\
& =\frac{1}{T_{U}-T_{L}} \int_{t \in T_{\mathrm{int}}}\left(m_{i}^{\{W\}}(t) m_{k}^{\{F\}}(t)+m_{i}^{\{F\}}(t) m_{k}^{\{W\}}(t)-m_{i}^{W}(t) m_{k^{\prime}}^{\{F\}}(t)-m_{i}^{\{F\}}(t) m_{k^{\prime}}^{\{W\}}(t)\right) d t \\
& =\frac{1}{T_{U}-T_{L}} \int_{t \in T_{\mathrm{int}}}\left(m_{i}^{\{W\}}(t)\left(m_{k}^{\{F\}}(t)-m_{k^{\prime}}^{\{F\}}(t)\right)+m_{i}^{\{F\}}(t)\left(m_{k}^{\{W\}}(t)-m_{k^{\prime}}^{\{W\}}(t)\right)\right) d t \\
& <0
\end{aligned}
$$

Proof of Property 7: The associative property of $J D\left(\mathbf{m}_{i}, \mathbf{m}_{j}\right)$ can be proved by:

$$
\begin{aligned}
& J D\left(\mathbf{m}_{i}, \mathbf{m}_{j}\right)=\frac{1}{T_{U}-T_{L}} \int_{t \in T_{\mathrm{int}}} J D\left(\mathbf{m}_{i}(t), \mathbf{m}_{j}(t)\right) d t \\
& =\frac{1}{T_{U}-T_{L}} \int_{t \in T_{\mathrm{int}}} \sqrt{\frac{1}{2}\left(\mathbf{m}_{i}(t)-\mathbf{m}_{j}(t)\right) \mathbf{J a c}\left(\mathbf{m}_{i}(t)-\mathbf{m}_{j}(t)\right)^{T}} d t \\
& =\frac{1}{T_{U}-T_{L}} \int_{t \in T_{\mathrm{int}}} \sqrt{\frac{1}{2}\left(-\left(\mathbf{m}_{j}(t)-\mathbf{m}_{i}(t)\right)\right) \mathbf{J a c}\left(-\left(\mathbf{m}_{j}(t)-\mathbf{m}_{i}(t)\right)\right)^{T}} d t \\
& =\frac{1}{T_{U}-T_{L}} \int_{t \in T_{\mathrm{int}}} J D\left(\mathbf{m}_{j}(t), \mathbf{m}_{i}(t)\right) d t \\
& =J D\left(\mathbf{m}_{j}, \mathbf{m}_{i}\right)
\end{aligned}
$$

Proof of Property 8: The average Jousselme distance can be extended as:

$$
\begin{aligned}
& J D\left(\mathbf{m}_{i}, \mathbf{m}_{j}\right)=\frac{1}{T_{U}-T_{L}} \int_{t \in T_{\text {int }}} J D\left(\mathbf{m}_{i}(t), \mathbf{m}_{j}(t)\right) d t \\
& =\frac{1}{T_{U}-T_{L}} \int_{t \in T_{\text {Tim }}} \sqrt{\frac{1}{2}\left(\mathbf{m}_{i}(t)-\mathbf{m}_{j}(t)\right) \mathbf{J a c}\left(\mathbf{m}_{i}(t)-\mathbf{m}_{j}(t)\right)^{T}} d t \\
& =\frac{1}{T_{U}-T_{L}} \int_{t \in T_{\text {Tim }}} \sqrt{\frac{1}{2}\left(\left\|\mathbf{m}_{i}(t)\right\|^{2}+\left\|\mathbf{m}_{j}(t)\right\|^{2}-2 \sum_{A \in \Omega} \sum_{B \subset \Omega} m_{1}^{A}(t) m_{2}^{B}(t) \frac{|A \cap B|}{|A \cup B|}\right.} d t
\end{aligned}
$$

where $\left\|\mathbf{m}_{i}(t)\right\|^{2}$ is the square norm of $\mathbf{m}_{i}(t)$. Suppose that $\mathbf{m}_{j}$ is a vacuous BBA, i.e., $\mathbf{m}_{j}=(0,0,0,1)$, then we have $\left\|\mathbf{m}_{j}(t)\right\|^{2}=1$ and $B=\Omega$, Eq. (B.2) can be further expanded as

$$
\begin{aligned}
J D\left(\mathbf{m}_{i}, \mathbf{m}_{j}\right) & =\frac{1}{T_{U}-T_{L}} \int_{t \in T_{\text {int }}} \sqrt{\frac{1}{2}\left(1+\left\|\mathbf{m}_{i}(t)\right\|^{2}-\sum_{A \subset \Omega} m_{i}^{A}(t)|A|\right)} d t \\
& =\frac{1}{T_{U}-T_{L}} \int_{t \in T_{\text {int }}} \sqrt{\frac{1}{2}\left(\left\|\mathbf{m}_{i}(t)\right\|^{2}-m_{i}^{\{W, F\}}(t)\right)} d t \\
& \geq 0
\end{aligned}
$$

The equality of Eq. (B.3) holds if and only if $\mathbf{m}_{i}$ is also a vacuous BBA, that is $\left\|\mathbf{m}_{i}(t)\right\|^{2}=m_{i}^{\{W, F\}}(t)=1$ at all time $t$. The proof is completed.

\section{REFERENCES}

[1] Y. Liu, B. Zhang, T. Jiang, T. Xiahou, "Optimization of multilevel inspection strategy for nonrepairable multistate systems", IEEE Transactions on Reliability, vol. 69, no. 3, pp. 968-985, 2020.
[2] S. Qiu, Henry X. G. Ming, "Reliability evaluation of multi-state seriesparallel systems with common bus performance sharing considering transmission loss", Reliability Engineering \& System Safety, vol. 189, pp. 406-415, 2019.

[3] H. Xiao, R. Peng, "Optimal allocation and maintenance of multi-state elements in series-parallel systems with common bus performance sharing", Computers \& Industrial Engineering, vol. 72, pp. 143-151, 2014.

[4] S. Destercke, M. Sallak, "An extension of universal generating function in multi-state systems considering epistemic uncertainties", IEEE Transactions on Reliability, vol. 62, no. 2, pp. 504-514, 2013.

[5] Y. Mo, L. Xing, S. V. Amari, "A multiple-valued decision diagram-based method for efficient reliability analysis of non-repairable phased-mission systems", IEEE Transactions on Reliability, vol. 63, no. 1, pp. 320-330, 2014.

[6] Z. Xu, Y. Mo, Y. Liu, T. Jiang, "Reliability assessment of multi-state phased-mission systems by fusing observation data from multiple phases of operation”, Mechanical Systems \& Signal Processing, vol. 118, pp. 603-622, 2019.

[7] Z. Zeng, S. Du, Y. Ding, "Resilience analysis of multi-state systems with time-dependent behaviors", Applied Mathematical Modelling, 2020. Doi.org/10.1016/j.apm.2020.08.066.

[8] M. Hathout, M. Vuillet, C. Carvajal, L. Peyras, Y. Diab, "Expert judgments calibration and combination for assessment of river levee failure probability", Reliability Engineering \& System Safety, vol. 188, pp. 377-392, 2019.

[9] W. Peng, H. Z. Huang, M. Xie, Y. J. Yuan, Y. Liu, “A Bayesian approach for system reliability analysis with multilevel pass-fail, lifetime and degradation data sets", IEEE Transactions on Reliability, vol. 62, no. 3, pp. 689-699, 2013.

[10] T. Xiahou, Y. Liu, "Reliability bounds for multi-state systems by fusing multiple sources of imprecise information", IISE Transactions, vol. 52, no. 9, pp. 1014-1031,2020.

[11] T. Xiahou, Y. Liu, T. Jiang, "Extended composite importance measures for multi-state systems with epistemic uncertainty of state assignment", Mechanical Systems \& Signal Processing, vol. 109, pp. 305-329, 2018.

[12] T. Xiahou, Z. Zeng, Y. Liu, "Remaining useful life prediction by fusing experts' knowledge and condition monitoring information", IEEE Transactions on Industrial Informatics, 2020. DOI: 10.1109/TII.2020. 2998102.

[13] Y. Ding, A. Lisnianski, "Fuzzy universal generating functions for multistate system reliability assessment", Fuzzy Sets \& Systems, vol. 159, no. 3, pp. 307-324, 2008.

[14] Y. Liu, H. Z. Huang, "Reliability assessment for fuzzy multi-state systems", International Journal of Systems Science, vol. 41, no. 4, pp. 365-379, 2010

[15] C. Li, X. Chen, X. Yi, J. Y. Tao, "Interval-valued reliability analysis of multi-state systems", IEEE Transactions on Reliability, vol. 60, no. 1, pp. 323-330, 2011.

[16] M. Sallak, W. Schön, F. Aguirre, "Reliability assessment for multi-state systems under uncertainties based on the Dempster-Shafer theory", IIE Transactions, vol. 45, no. 9, pp. 995-1007, 2013.

[17] G. M. Galante, C. M. La Fata, T. Lupo, P. Gianfranco, "Handling the epistemic uncertainty in the selective maintenance problem", Computers \& Industrial Engineering, vol. 141, pp. 106293, 2020.

[18] W. Liu, "Analyzing the degree of conflict among belief functions", Artificial Intelligence, vol. 170, pp. 909-924, 2006. 
[19] A. P. Dempster, "Upper and lower probabilities induced by a multivalued mapping", The Annals of Mathematical Statistics, vol. 38, pp. 325-339, 1967.

[20] G. Shafer, "A Mathematical Theory of Evidence", Princeton University Press, 1976.

[21] J. Schubert, "Conflict management in Dempster-Shafer theory using the degree of falsity", International Journal of Approximate Reasoning, vol. 52, no. 3, pp. 449-460, 2011.

[22] S. Destercke, T. Burger, "Toward an axiomatic definition of conflict between belief functions", IEEE Transactions on Cybernetics, vol. 43, no. 2, pp. 585-596, 2013

[23] A. L. Jousselme, D. Grenier, É. Bossé, "A new distance between two bodies of evidence", Information Fusion, vol. 2, no. 2, pp. 91-101, 2001.

[24] F. Cuzzolin, "A geometric approach to the theory of evidence", IEEE Transactions on Systems, Man, and Cybernetics, Part C Applications and Reviews, vol. 38, no. 4, pp. 522-534, 2008.

[25] A. L. Jousselme, P. Maupin, "Distances in evidence theory: Comprehensive survey and generalizations", International Journal of Approximate Reasoning, vol. 53, no. 2, pp. 118-145, 2012.

[26] S. Bi, M. Broggi, M. Beer, "The role of the Bhattacharyya distance in stochastic model updating”, Mechanical Systems \& Signal Processing, vol. 117, pp. 437-452, 2019.

[27] Q. Zhang, R. Kang, M. Wen. Belief reliability for uncertain random systems. IEEE Transactions on Fuzzy Systems, vol 26, no. 6, pp. 36053614, 2018.

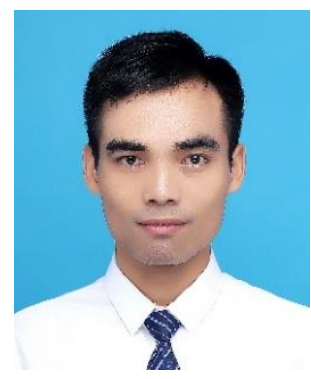

Tangfan Xiahou received his B.E. degrees in Industrial Engineering from the University of Electronic Science and Technology of China in 2015 and the M.S. degree in Mechanical Engineering from UESTC in 2018. He is currently a $\mathrm{PhD}$ student in the School of Mechanical and Electrical Engineering at UESTC. His research interest includes reliability modeling under uncertainty, Dempster-Shafer evidence theory, and prognostics and health management (PHM).

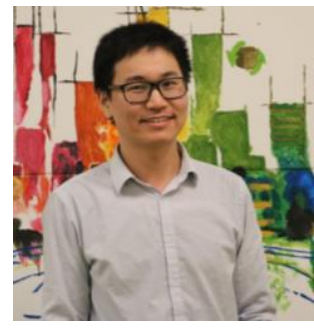

Zhiguo Zeng received the Ph.D. degree in reliability engineering from Beihang university in 2016. He is currently an assistant professor at CentraleSupélec, Université Paris-Saclay, France. His research focuses on the characterization and modeling of the failure/repair/maintenance behavior of components, complex systems and their reliability, maintainability, prognostics, safety, vulnerability and security. Dr. ZENG is an author/co-author of more than 50 papers in highly recognized international journals and conferences (including 26 journal papers indexed in Web of Science).
[28] R. M. Cooke, L. H. J. G. Louis, "TU Delft expert judgment data base", Reliability Engineering \& System Safety, vol. 93, no. 5, pp. 657-674, 2008.

[29] T. Xiahou, Y. Liu, Q. Zhang, "Multi-objective redundancy allocation for multi-state system design under epistemic uncertainty of component states", ASME Journal of Mechanical Design, vol. 142, no. 11, pp. $111703,2020$.

[30] B. Ristic, P. Smets, "The TBM global distance measure for the association of uncertain combat ID declarations", Information Fusion, vol. 7, no. 3, pp. 276-284, 2006.

[31] A. Ayoun, P. Smets. Data association in multi-target detection using the transferable belief model. International Journal of Intelligent Systems, vol 16, no. 10, pp. 1167-1182, 2001.

[32] M. Bouchard, A. P. Jousselme, P. E. Doré, "A proof for the positive definiteness of the Jaccard index matrix", International Journal of Approximate Reasoning, vol. 54, no. 5, pp. 615-626, 2013.

[33] E. Choi, C. Lee, "Feature extraction based on the Bhattacharyya distance", Pattern Recognition, vol. 36, no. 8, pp. 1703-1709, 2003.

[34] O. Michailovich, Y. Rathi, A. Tannenbaum, "Image segmentation using active contours driven by the Bhattacharyya gradient flow", IEEE Transactions on Image Processing, vol. 16, no. 11, pp. 2787-2801, 2007.

[35] C. H. You, K. A. Lee, H. Li, "An SVM kernel with GMM-supervector based on the Bhattacharyya distance for speaker recognition", IEEE Signal Processing Letters, vol. 16, no. 1, pp. 49-52, 2008.

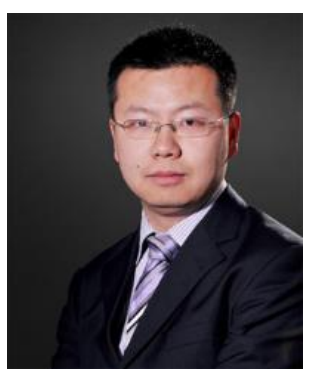

Yu Liu (M'14, SM'2018) is a full Professor in the School of Mechanical and Electrical Engineering, at the University of Electronic Science and Technology of China. He received his PhD degree in Mechatronics Engineering from the University of Electronic Science and Technology of China. He was a Visiting Pre-doctoral Fellow in the Department of Mechanical Engineering at Northwestern University, U.S.A. from 2008 to 2010 and a Postdoctoral Research Fellow in the Department of Mechanical Engineering, at the University of Alberta, Canada from 2012 to 2013. He has published over 60 peer-reviewed papers in international journals and conferences. His research interests include system reliability modeling and analysis, maintenance decisions, prognostics and health management, and design under uncertainty.

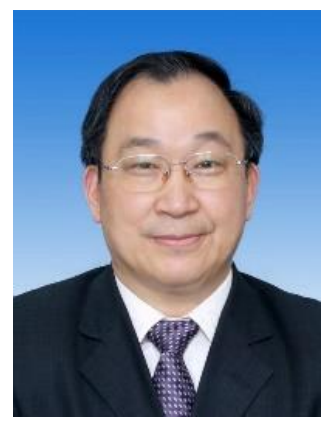

Hong-Zhong Huang is a full Professor of Mechanical Engineering with the School of Mechanical and Electrical Engineering, University of Electronic Science and Technology of China, Chengdu, China. He is also the Director of the Center for System Reliability and Safety, University of Electronic Science and Technology of China. He has held visiting appointments at several universities in the USA, Canada, and Asia. He has authored or coauthored more than 280 journal papers and eight books in the fields of reliability engineering, optimization design, fuzzy sets theory, and product development. Prof. Huang is also an ISEAM Fellow, a Technical Committee Member of ESRA, a Co-Editor-in-Chief for the International Journal of Reliability and Applications, and an Editorial Board Member of several international journals. 
